\title{
Engineering Silk Fibroin-Based Nerve Conduit with Neurotrophic Factors for Proximal Protection after Peripheral Nerve Injury
}

\author{
Cristiana R. Carvalho, Wei Chang, Joana Silva-Correia, Rui L. Reis, Joaquim M. Oliveira,* \\ and Joachim Kohn
}

\begin{abstract}
Artificial nerve conduits capable of adequately releasing neurotrophic factors are extensively studied to bridge nerve defects. However, the lack of neurotrophic factors in the proximal area and their visible effects in axonal retrograde transport following nerve injury is one of the factors causing an incomplete nerve regeneration. Herein, an advanced conduit made of silk fibroin is produced, which can incorporate growth factors and promote an effective regeneration after injury. For that, enzymatically crosslinked silk fibroin-based conduits are developed to be used as a platform for the controlled delivery of neurotrophic factors. Nerve growth factor and glial-cell line derived neurotrophic factor (GDNF) are incorporated using two different methodologies: i) crosslinking and ii) absorption method. The release profile is measured by ELISA technique. The bioactivity of the neurotrophic factors is evaluated in vitro by using primary dorsal root ganglia. When implanted in a $10 \mathrm{~mm}$ sciatic nerve defect in rats, GDNF-loaded silk fibroin conduits reveal retrograde neuroprotection as compared to autografts and plain silk fibroin conduit. Therefore, the novel design presents a substantial improvement of retrograde trafficking, neurons' protection, and motor nerve reinnervation.
\end{abstract}

surgically repairing PNIs, autologous nerve graft is the current gold standard option for bridging gaps that cannot be repaired by direct suturing. Despite that, the autograft is often associated with increased morbidity, painful neuroma formation, and inappropriate match of nerves. ${ }^{[2]}$ To solve some of the problems related to biological grafts, artificial nerve guidance conduits (NGCs) have been developed as an alternative strategy. ${ }^{[2]}$ The properties of the biopolymer used to engineer NGCs and the features of the final conduit itself, such as pore size and degradation, play a crucial role in the nerve regeneration efficiency. The biomaterial must be biocompatible and support cellular growth, proliferation, and migration. ${ }^{[3]}$ Furthermore, NGCs must allow the passage of nutrients and oxygen, but at the same time, inhibit the infiltration of fibroblasts, which may form scar tissue at the injury site. ${ }^{[4]}$ Mechanical properties that support nerve regeneration and flexibility

\section{Introduction}

Peripheral nerve injuries (PNIs) are one of the most important burdens in public healthcare systems worldwide. They are considered intractable and a common disease with a high and gradually increasing incidence over the past decades. ${ }^{[1]}$ When

Dr. C. R. Carvalho, Dr. J. Silva-Correia, Prof. R. L. Reis, Dr. J. M. Oliveira 3B's Research Group-Biomaterials

Biodegradables and Biomimetics

Headquarters of the European Institute of Excellence on Tissue

Engineering and Regenerative Medicine

University of Minho

Barco, Guimarães 4805-017, Portugal

E-mail: miguel.oliveira@i3bs.uminho.pt

Dr. W. Chang, Prof. J. Kohn

New Jersey Center for Biomaterials

Rutgers

The State University of New Jersey

Piscataway, NJ 08854, USA

The ORCID identification number(s) for the author(s) of this article can be found under https://doi.org/10.1002/adhm.202000753

DOI: 10.1002/adhm.202000753 are also a prerequisite. ${ }^{[5]}$ The previously developed silk-fibroin (SF) conduits presented several advantages and overcame all these problems, showing features such as kinking resistanceability and suturability. ${ }^{[3]}$ Furthermore, silk-fibroin has had an important role in terms of peripheral nerve regeneration (PNR) over the last decades, and many successful strategies have been developed to address PNR hurdles. ${ }^{[6]}$ Being an inexpensive and natural occurring fibrous protein, this FDA-approved biomaterial possesses adequate physicochemical as well as mechanical properties for scaffolds production. Being an extremely versatile and biocompatible material, several groups published their experimental work, claiming it is an almost perfect biomaterial for PNR. ${ }^{[7]}$ Additionally, the process utilized in this experimental work contains only natural, biological and nontoxic components. Also, due to the natural silk properties, NTFs can be slowly released, showing very impressive long-term release.

However, even with the efforts and advances in the tissue engineering (TE) field and the fact that peripheral nervous system (PNS) has an intrinsic capacity to regenerate, ${ }^{[8]}$ poor outcomes are still a problem. They are mainly related to: I) death of primary motor and sensory neurons; II) absence of fiber regeneration over the gap area, and III) target-organ incomplete reinnervation, limiting the full recovery of the patients at a functional 
and sensory level. ${ }^{[9]}$ In fact, even when injured nerves are surgically restored in the optimal timeframe after the injury, the period that axons take to regenerate is too extended, resulting in the lack of contact between neurons and target organs. The consequence is a state of chronic axotomy and end targets become chronically denervated. ${ }^{[10]}$ Such problem is not solved by the use of simple hollow NGCs, that are FDA-approved and used extensively in the clinics, but only provide regenerating nerves with poor guidance and protection. ${ }^{[11]}$ Several strategies have been used to enhance functional and sensory recovery, aiming to increase the number of suitable axons which correctly reach their targets. In this sense, one of the most promising and prominent possibilities to improve nerve regeneration is the inclusion of neurotrophic factors (NTFs) in the NGCs. NTFs are endogenous proteins capable of binding to cell receptors with the purpose of modulating and directing cellular activities. ${ }^{[10]}$ In relation to the PNS, NTFs are able to promote neuronal survival, axonal regeneration and Schwann cells' migration. Furthermore, it has been reported that NTFs have a very good efficiency in enhancing not only neurogenesis when applied concomitantly with scaffolds, but also angiogenesis. ${ }^{[12]}$

With distinctive properties and functions, nerve growth factor (NGF) and glial cell line-derived neurotrophic factor (GDNF) were used. Briefly, NGF promotes survival and axonal outgrowth of sensory neurons both in vitro and in vivo, ${ }^{[13]}$ and has been proved to promote early axonal regeneration. However, the role of NGF in the recovery of motor neuron function is restricted. ${ }^{14]}$ On the other hand, GDNF is one of the most protective factors for motor neurons and is abundantly expressed by skeletal muscle. ${ }^{[15]}$ Nevertheless, it has also marked activity on sensory neurons. Acting through different intrinsic mechanisms and different pathways, both NTFs will have various effects on neurons and respective neurites, such as neurite elongation or density increase. In the case of NGF, the trkA receptor is a $140 \mathrm{kDa}$ glycoprotein which binds NGF. The NGF-activated trkA receptor undergoes dimerization and autophosphorylation at several tyrosine residues that selectively trigger activity in several intracellular signaling pathways, having direct action on sensory neurons. ${ }^{[16]}$ The GDNF, a neuronal survival factor, binds its coreceptor GDNF family receptor alpha1 (GFR alpha 1) and signals through the receptor tyrosine kinase RET, showing its effects on both sensory and motor neurons. These were the NTFs chosen for this experimental work, due to their distinctive properties.

In addition to the selection of NTFs, it is recognized that the selection of a suitable delivery method for these molecules is crucial for an effective regenerative potential. The inclusion and consequent deliver of growth promoting biological molecules can be achieved by several ways, accounting for many different and adjustable delivery systems. ${ }^{[10]}$ When considering the delivery of NTFs from the conduit wall, methods such as conjugation, ${ }^{[17]}$ adsorption, ${ }^{[18]}$ and physical entrapment in the wall ${ }^{[19]}$ are among the most used methods. Regardless of the delivery method, general complications related to loss of bioactivity, initial burst release or too low daily dose must be avoided in order to have a beneficial effect on nerve regeneration. ${ }^{[10]}$

Optimized delivery methods for NTFs eluted from SF conduits described in the literature are solely based in the simple blending of NTFs in SF solution without further entrapment or electrospinning technique. ${ }^{[6,20]}$
In this work, we have tested two methods: i) crosslinking and ii) adsorption, and compared their efficacy for retention and controlled delivery of GDNF or NGF. The methods are based on and interrelated with the SF NGC production method. For such, a new patented enzymatically crosslinked SF (eSF) conduit previously developed by our group was used. ${ }^{[3]}$ The crosslinking process utilizes tyrosine groups from the SF structure to form a hydrogel. Therefore, we hypothesized that during crosslinking, the NTFs would be trapped in a more complex and tighter SF matrix, ${ }^{[3]}$ creating a more appropriate condition for sustained release.

Such method was compared to the absorbing process, where the eSF conduits are immersed in a NTFs solution, in which, processes like absorption and adsorption were expected to occur. Due to the porosity of the conduit and its high surface-tovolume ratio for solution penetration, we could envision that the NTFs would be highly retained in the SF conduit via the second approach. Furthermore, the suitability of eSF to be processed in aqueous media, turns it a promising biomaterial for the fabrication of bioactive protein delivery systems. ${ }^{[21]}$

Since the importance of NTFs to enhance PNR was noticed, it has been investigated the importance of their retrograde transport to the cell body. ${ }^{[22]}$ A body of evidence proposes that the mechanism of NTF retrograde transport is essential for signal propagation, neuronal development, and prevention of neuronal degeneration. In the case of PNIs, the retrograde transport of injury signals is one of the indispensable cellular instruments leading to regeneration. ${ }^{[23]}$ This mechanism can allow the coordination between the injury site and the cell body, therefore regulating the proper genes to promote neuronal survival. Both NGF and GDNF have shown to be retrogradely transported, ${ }^{[22 b]}$ with beneficial effects in terms of nerve protection and regeneration, although with different mechanisms. ${ }^{[24]}$

The aim of this investigation was to assess the feasibility of retrograde delivery of GDNF or NGF by eSF conduits, with distinct release kinetics after different incorporation methods. The GDNF and NGF biological activities were assessed in vitro with a primary dorsal root ganglia explants (DRGs) model, after their direct seeding in Matrigel. The most promising formulation of NTF-loaded eSF conduit was then implanted in a $10 \mathrm{~mm}$ rat sciatic nerve injury model and compared to both autograph and eSF conduit. The $10 \mathrm{~mm}$ sciatic nerve injury model was used in order to create a gap to diminish the effect of distal neurotrophic factors, and in fact, assess the outcome of the NGCs delivered NTFs. The data were evaluated based on pinch test, muscle weight, and proximal nerve histology following sciatic nerve injury.

\section{Results}

\subsection{Production and Physical Characterization of Silk Fibroin Conduits}

SF conduits were successfully produced according to a protocol previously developed by our group. ${ }^{[3]}$ Briefly, a purified SF solution was diluted to $16 \mathrm{wt} \%$ with distilled water, after which it was mixed with horseradish peroxidase solution and hydrogen peroxide solution for the crosslink. The previous mixture was then injected within the space between two concentric cylinder molds. The system was incubated at $37^{\circ} \mathrm{C}$ to induce gelation for the 
A)

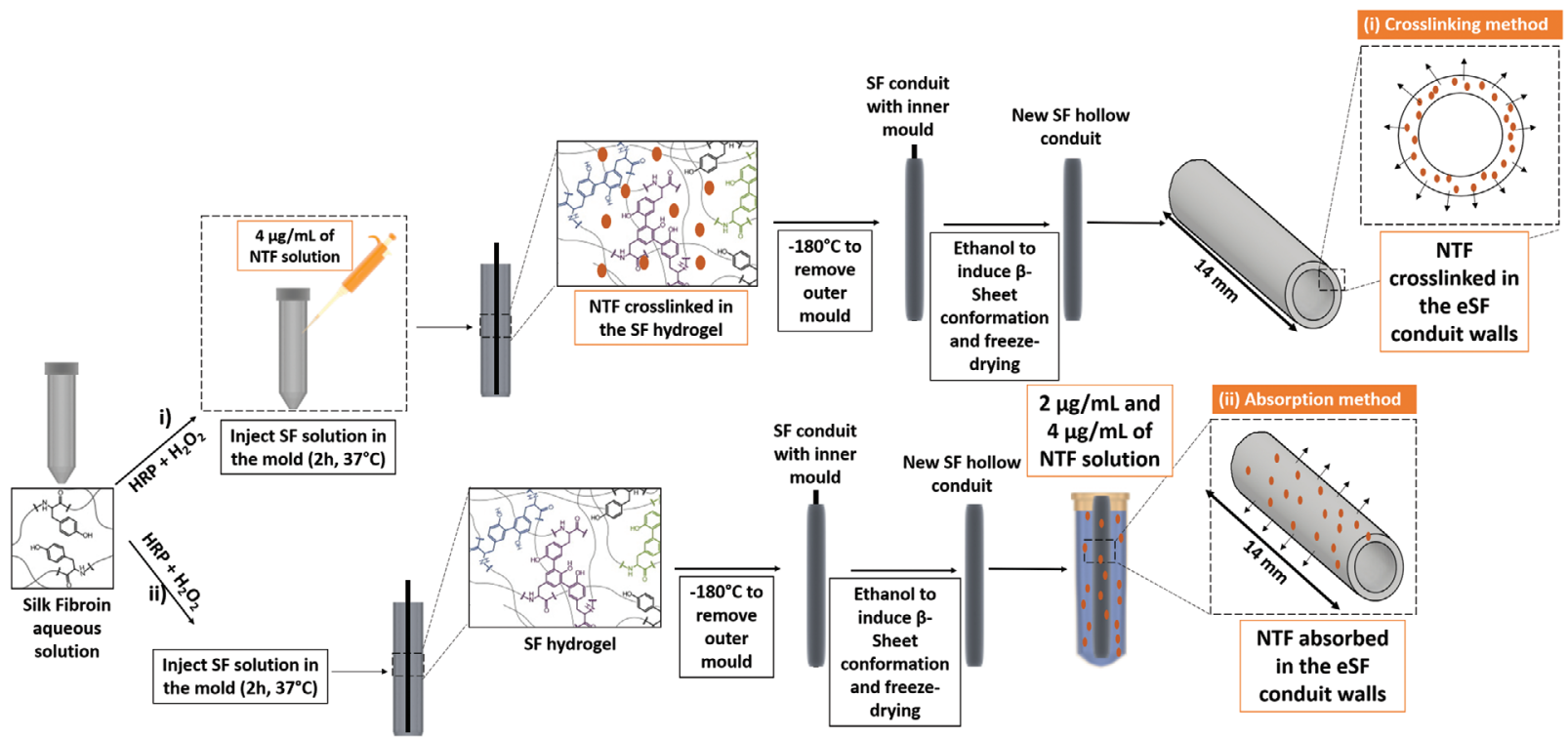

B)

I)

II)

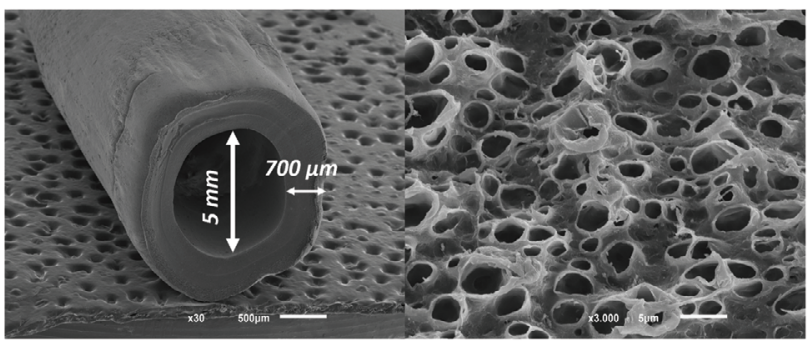

Figure 1. A) Schematic representation of the methodology used to produce the SF conduits. The methodology allows to fabricate enzymatically crosslinked SF NGCs following a method previously developed by Carvalho et al., which takes advantage of the tyrosine groups present in the structure of SF that are known to form a covalently crosslinked hydrogel. B) SEM microphotographs of the conduits incorporating NTFs, by (i) the crosslinking and (ii) the absorption method, respectively.

Table 1. Samples produced for in vitro assays by means of using the crosslinking and absorption methods, different NTFs (i.e., GDNF and NGF), and different NTFs concentrations (i.e., 2 and $4 \mu \mathrm{g} \mathrm{mL}^{-1}$ ).

\begin{tabular}{|c|c|c|c|}
\hline & $\begin{array}{l}\text { Crosslinking } \\
4 \mathrm{\mu g} \mathrm{mL}^{-1}\end{array}$ & $\begin{array}{l}\text { Absorption } \\
2 \mu \mathrm{g} \mathrm{mL}-1\end{array}$ & $\begin{array}{l}\text { Absorption } \\
4 \mathrm{\mu g} \mathrm{mL}^{-1}\end{array}$ \\
\hline GDNF & GDNF/C & GDNF/A $A_{2}$ & $\mathrm{GDNF} / \mathrm{A}_{4}$ \\
\hline NGF & $\mathrm{NGF} / \mathrm{C}$ & $\mathrm{NGF} / \mathrm{A}_{2}$ & $\mathrm{NGF} / \mathrm{A}_{4}$ \\
\hline
\end{tabular}

period of 30 min. After gelation, a quick immersion in liquid nitrogen is needed, followed by an immersion in ethanol to induce permanent $\beta$-sheet.

Figure 1 depicts a detailed scheme of the production steps, in which the different methods of NTFs incorporation are represented. Different formulations were produced, by varying the incorporation method (i.e., crosslinking and absorption), the NTF incorporated (i.e., NGF and GDNF) and the concentration of NTFs (i.e., $2 \mu \mathrm{g} \mathrm{mL} L^{-1}$ or $4 \mu \mathrm{g} \mathrm{mL} L^{-1}$ ), as can be seen in Table 1.

The microstructure of the final conduits is shown in Figure S1 (Supporting Information), which shows representative SEM micrographs of the eSF NGCs microstructure.

\subsection{Neurotrophic Factors Loading Efficiency}

NTFs loading in the eSF conduits was attained by two different methods designated by crosslinking and absorption.

In the crosslinking method, $4 \mu \mathrm{g} \mathrm{mL}^{-1}$ of each NTF was incorporated in $1 \mathrm{~mL}$ of $16 \% \mathrm{SF}$ solution. The rest of the process was carried out according to the previous report ${ }^{[3]}$ and was shown in Figure 1, to finally obtain a freeze-dried eSF NGC. For GDNF and NGF crosslinked formulations, the loading efficiency can be considered $100 \%$, as all the amount of growth factor that was added in the $4 \mu \mathrm{g} \mathrm{mL}^{-1}$ solution got entrapped in SF structure.

However, the loading efficiency in the absorption method needed to be determined. For that, ELISA quantification was performed on the remaining NTF solution after the absorption process, in order to calculate the amount of growth factor that was, in fact, absorbed. In Table 2, the loading efficiency determined for the different formulations is presented. For GDNF/ $\mathrm{A}_{2}$ formulation, the loading efficiency reached $78.3 \pm 1.25 \%$. A similar value $(77.2 \pm 0.85 \%)$ was determined for $\mathrm{GDNF} \mathrm{A}_{4}$ formulation. The retention of NGF by the eSF conduit was inferior when compared to GDNF, as the loading efficiency of $46.2 \pm 0.43 \%$ and $44.5 \pm$ $1.02 \%$ was observed for $\mathrm{NGF} / \mathrm{A}_{2}$ and $\mathrm{NGF} / \mathrm{A}_{4}$, respectively. 
Table 2. Loading efficiency verified for the different formulations, shown in percentage.

\begin{tabular}{lc}
\hline Formulation & Loading efficiency \\
\hline $\mathrm{GDNF} / \mathrm{A}_{2}$ & $78.3 \pm 1.25 \%$ \\
$\mathrm{GDNF} / \mathrm{A}_{4}$ & $77.2 \pm 0.85 \%$ \\
$\mathrm{NGF} / \mathrm{A}_{2}$ & $46.2 \pm 0.43 \%$ \\
$\mathrm{NGF} / \mathrm{A}_{4}$ & $44.5 \pm 1.02 \%$
\end{tabular}

\subsection{In Vitro Release of NTFs from Silk Conduit}

The amount of released GDNF or NGF from the eSF conduits was quantified daily within the first $5 \mathrm{~d}$ and in a cumulative manner up to $50 \mathrm{~d}$, according to the different methods of loading and concentrations of NTFs (Figure 2). The scheme of the methodology used in the NTF release study is depicted in Figure 2A.
The daily GDNF release profile presented in Figure 2B-I reveals a significantly higher delivery of the NTF when the loading is done by means of performing the absorption method as compared to crosslinking method, in all the timepoints. Regarding the absorption formulations, the highest released was verified in the first day, with an average of $2.45 \pm 0.42 \mathrm{ng} \mathrm{mL}-1$ of released NTF. It was also verified a reduction of the delivery rate day by day, from $2.45 \pm 0.42 \mathrm{ng} \mathrm{mL}-1$ on day 1 to $1.06 \pm 0.12 \mathrm{ng} \mathrm{mL}-1$ on day 5 (GDNF/A formulation).

With a slightly overall lower release, there was also a reduction from $2.19 \pm 0.15 \mathrm{ng} \mathrm{mL} \mathrm{m}^{-1}$ in day 1 to $1.04 \pm 0.12 \mathrm{ng}$ $\mathrm{mL}^{-1}$ in day 5 for GDNF/A formulation. The cumulative release shown in Figure 2B-II reveals that the tendency to slowly decrease the amount of NTF released is kept throughout the timepoints, with GDNF/ $\mathrm{A}_{4}$ formulation releasing $36.8 \pm 0.9 \mathrm{ng} \mathrm{mL}-1$ and $\mathrm{GDNF} / \mathrm{A}_{2}$ releasing $32.3 \pm 3.3 \mathrm{ng} \mathrm{mL} \mathrm{m}^{-1}$ after $50 \mathrm{~d}$. The daily NGF releasing profile can be seen in Figure 2B-III where, similarly to GDNF daily release, the higher amount released was verified in

A)

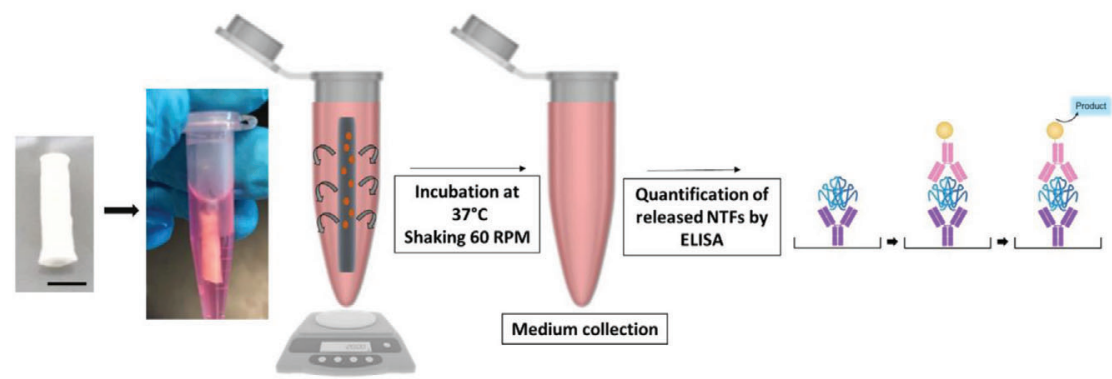

B)

GDNF

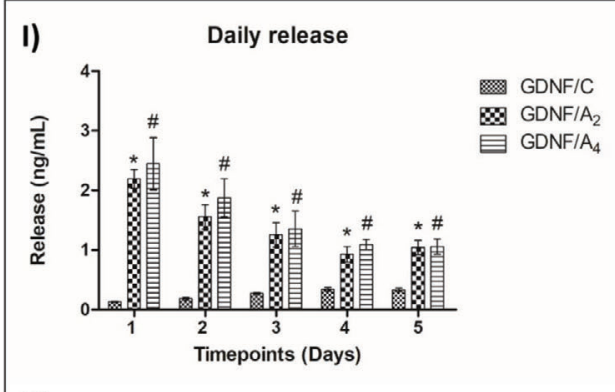

II)

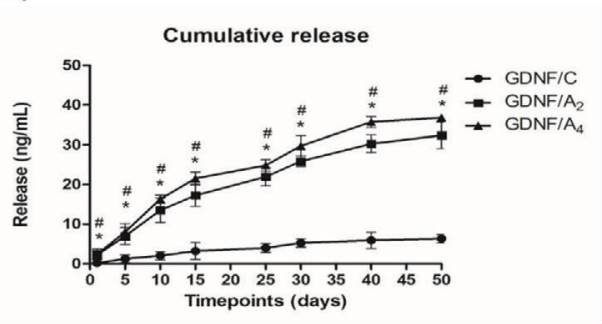

NGF

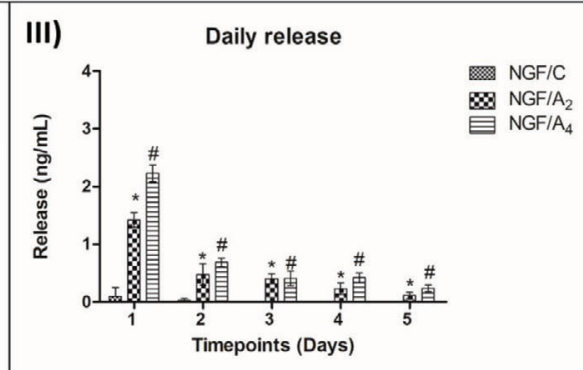

IV)

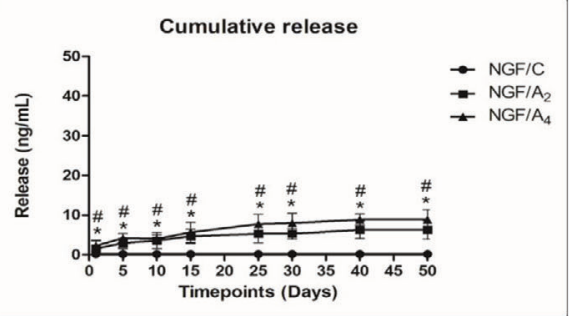

Figure 2. A) Scheme of the methodology used in the NTF release assay. B) ELISA quantification of the NTFs released from the SF conduit, considering the different methods of incorporation, NTFs used and concentration. B-I) Daily amount of GDNF released from SF conduits within 5 d; B-II) Cumulative amount of released GDNF within 50 d; B-III) Daily amount of NGF released from SF conduits within 5 d; B-IV) Cumulative amount of released NGF within $50 \mathrm{~d}$. Results were represented as mean $\pm \mathrm{SD}(n=3)$. For multiple comparisons, * symbol indicates the comparison of GDNF/A $\mathrm{Or}_{2} \mathrm{NGF} / \mathrm{A}_{2}$ and crosslinked formulations, \# symbol regards comparison of GDNF/A $\mathrm{A}_{4}$ or NGF/A and crosslinked formulations. Statistically significant differences were represented by $* / \#(p<0.05), * * / \# \#(p<0.01)$, and $* * * * \# \# \#(p<0.001)$. 
the first day, but with a more drastic reduction from day 1 to day 2 , in a burst release manner. $\mathrm{NGF} / \mathrm{A}_{4}$ formulation reduced its release from $2.23 \pm 0.14 \mathrm{ng} \mathrm{mL} \mathrm{m}^{-1}$ in day 1 to $0.24 \pm 0.05 \mathrm{ng} \mathrm{mL}$ in day 5 , whereas $\mathrm{NGF} / \mathrm{A}_{2}$ formulation reduced its release from $1.42 \pm 0.12 \mathrm{ng} \mathrm{mL} \mathrm{m}^{-1}$ on day 1 to $0.11 \pm 0.06 \mathrm{ng} \mathrm{mL}^{-1}$ in day 5 . The cumulative release of NGF depicted in Figure 2B-IV shows that the tendency to decrease NTF release is kept, with NGF/A formulation releasing $8.9 \pm 2.1 \mathrm{ng} \mathrm{mL}^{-1}$ and $\mathrm{NGF} / \mathrm{A}_{2}$ releasing $6.3 \pm 2.3 \mathrm{ng} \mathrm{mL}^{-1}$ after $50 \mathrm{~d}$. In all timepoints analyzed and for both GDNF and NGF, there is a significant statistical difference between the formulations produced using the absorption method in comparison to the crosslinked formulations.

When calculations are made in terms of percentage of release considering the loading efficiency of each formulation, an inverse tendency is observed regarding the amount of NTF that is incorporated. Figure S2 (Supporting Information) reveals a higher percentage of release when observing formulations $\mathrm{GDNF} / \mathrm{A}_{2}$ and $\mathrm{NGF} / \mathrm{A}_{2}$, as compared to GDNF/A $\mathrm{A}_{4}$ and NGF/A . A high amount of NTF remains in the conduits when $4 \mu \mathrm{g} \mathrm{mL}-1$ are incorporated.

\subsection{NTFs Bioactivity Assay with DRGs}

The responses of the DRGs when subjected to the different conditions and different NTFs were evaluated in terms of neurite outgrowth (Figure 3), after seeding in Matrigel. The scheme of the methodology used in the bioactivity assay in vitro is presented in Figure 3A. DRGs responses to GDNF can be observed qualitatively and quantitatively in Figure 3B,C, respectively. GDNF/A formulation presented the highest values for all the parameters, whereas the crosslinked formulation presented the lowest, even lower than the negative control. More specifically, in terms of neurite length, there is a significant difference between the crosslinked samples (GDNF/C) and both absorption formulations $\left(\mathrm{GDNF} / \mathrm{A}_{2}\right.$ and $\left.\mathrm{GDNF} / \mathrm{A}_{4}\right)$, where the longest neurites extended for an average of $122.8 \pm 119.3,595.2 \pm 246.2$, and $759.0 \pm$ $160.7 \mu \mathrm{m}$, respectively. For the positive control and negative control, neurite length achieved values of $961.7 \pm 197.8$ and $492.0 \pm$ $170.5 \mu \mathrm{m}$, respectively. Regarding cellular migration from the DRG body, there was no significant differences between the values obtained for all the tested groups. The values obtained for the tested formulations (GDNF/C, GDNF/A $\mathrm{A}_{2}$ and GDNF/A4), are $712.8 \pm 281.3 \mu \mathrm{m}, 1422.1 \pm 417.6 \mu \mathrm{m}$, and $1525.8 \pm 384.9 \mu \mathrm{m}$, respectively. For the positive and negative control, the values were $1307.3 \pm 266.9 \mu \mathrm{m}$ and $854.7 \pm 282.9 \mu \mathrm{m}$. In what concerns the percentage of field coverage, the values obtained were $1.3 \pm$ $0.8 \%, 4.4 \pm 1.5 \%$, and $10.9 \pm 1.6 \%$ for GDNF/C, GDNF/A 2 and $\mathrm{GDNF} / \mathrm{A}_{4}$ formulations, respectively. In the positive and negative controls, values of $15.6 \pm 5.3 \%$ and $3.1 \pm 1.3 \%$ were obtained, respectively. For this parameter, a statistically significant difference was observed for GDNF/A formulation when compared to $\mathrm{GDNF} / \mathrm{C}$ and GDNF/A $\mathrm{A}_{2}$ samples. A summary of the abovementioned information regarding GDNF treated DRGs can be found in Table S1 (Supporting Information).

DRGs responses to NGF can be seen qualitatively and quantitatively in Figure 3D,E, respectively. The results obtained with NGF followed the same pattern as with GDNF. The NGF/A formulation presented consistently higher values as compared to $\mathrm{NGF} / \mathrm{A}_{2}$ formulation, whereas lower neurite outgrowth was observed for NGF/C when compared to the negative control. More precisely, the neurite length reached values of $1072.6 \pm$ $227.4 \mu \mathrm{m}$ for the positive control and $492.0 \pm 170.5 \mu \mathrm{m}$ for the negative control. Regarding the formulations NGF/C, NGF/A and $\mathrm{NGF} / \mathrm{A}_{4}$, the determined values were $220.5 \pm 116.6,641.1 \pm$ 176.4 , and $870.9 \pm 246.7 \mu \mathrm{m}$, respectively. For this parameter, significant statistical differences were found between all the formulations containing NGF. For cellular migration, image analysis indicated a migration of $1464.8 \pm 262.9 \mu \mathrm{m}$ for the positive control and $854.7 \pm 282.9 \mu \mathrm{m}$ for the negative control. Regarding the formulations $\mathrm{NGF} / \mathrm{A}_{2}$ and $\mathrm{NGF} / \mathrm{A}_{4}$, the measured values were $1196.2 \pm 285.6$ and $1560.4 \pm 403.6 \mu \mathrm{m}$, respectively. In contrast, for $\mathrm{NGF} / \mathrm{C}$, no cellular migration was observed. A significant statistical difference was found between $\mathrm{NGF} / \mathrm{A}_{2}$ and $\mathrm{NGF} / \mathrm{A}_{4}$. For the percentage of field coverage obtained with NGF formulations, the values for the formulations $\mathrm{NGF} / \mathrm{C}, \mathrm{NGF} / \mathrm{A}_{2}$ and $\mathrm{NGF} / \mathrm{A}_{4}$ were $8 \pm 1.6 \%, 4.2 \pm 1.0 \%$, and $7 \pm 1.4 \%$, respectively. On the other hand, values of $7.0 \pm 1.3 \%$ and $3.1 \pm 1.3 \%$ were determined for the positive control and negative control, respectively. Significant statistical differences were found between the two NGF absorption formulations and between NGF/A $\mathrm{A}_{4}$ and $\mathrm{NGF} / \mathrm{C}$ formulation. A summary of above-mentioned information regarding NGF treated DRGs can be found in Table S2 (Supporting Information).

When comparing GDNF/A 4 with $\mathrm{NGF} / \mathrm{A}_{4}$ in terms of percentage of field coverage (Figure 4 ), which reflects both the length and density of neurite outgrowth, it was observed a statistically significant difference between them, with values of $10.9 \pm 1.6 \%$ and $7 \pm 1.4 \%$, respectively. Due to the statistically significant different results obtained between both NTFs, GDNF/A formulation was selected to proceed with further in vivo studies.

Taking a closer look at the confocal images, it is clear that the migratory stage of Schwann cells and the secretion of laminin cues closely interacts with axons (Figure S3, Supporting Information). ${ }^{[25]}$ It was also interesting to observe the axon physiology in the DRGs, where nucleated cells, assumed to be Schwann cells, were surrounding the growing neurites to form the myelin sheets. ${ }^{[26]}$ Another axon's typical structure, the terminal button, is visible in Figure S4 (Supporting Information), confirming the presence of growing axons.

\subsection{In Vivo Study}

Three different study groups were tested in vivo. The autograft, $\mathrm{eSF}$ and GDNF/A $\mathrm{A}_{4}$ were implanted in a $10 \mathrm{~mm}$ sciatic nerve defect in rats, by performing a sciatic nerve resection.

The 3D printed tool allowed for a very clean and precise cut of the sciatic nerve, in the exact required position. All experimental animals tolerated the anesthesia and surgery and survived until the end of the observation period. Throughout the 6 weeks of in vivo experiment, animals were checked at least once a day and no complications were observed. All rats recovered well, without any sign of infection in the wounds, which healed completely approximately one week after surgery.

No signs of ulcers or autophagy were verified in the paws of any of the 15 operated rats. Overall, no visible distress was detected in the animals after surgery. At the time of explantation, no signs 
A)
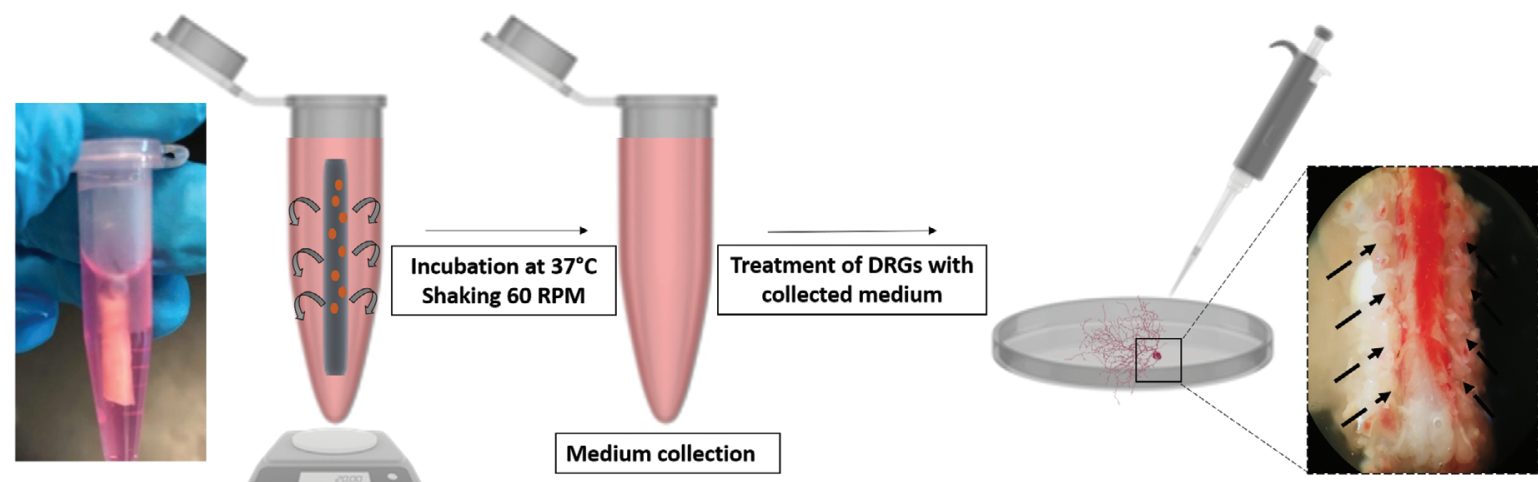

B)
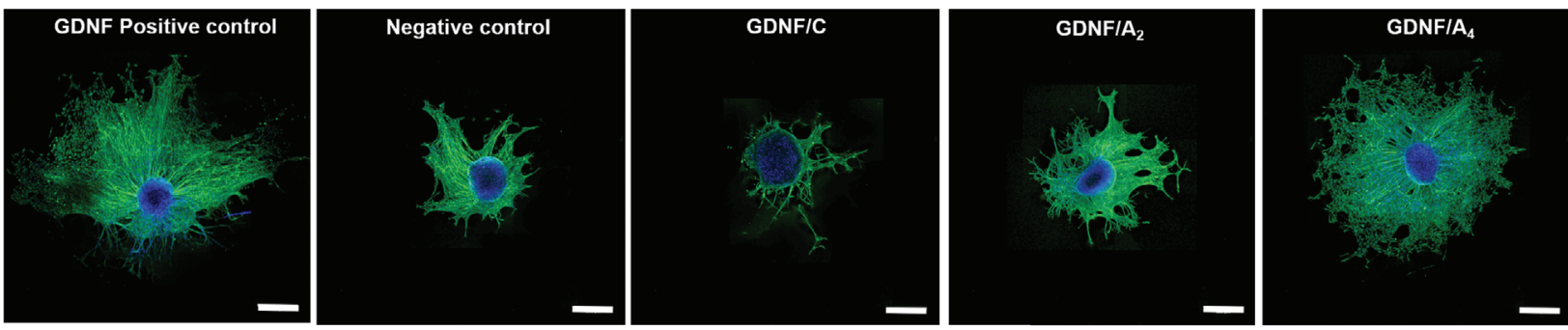

C)

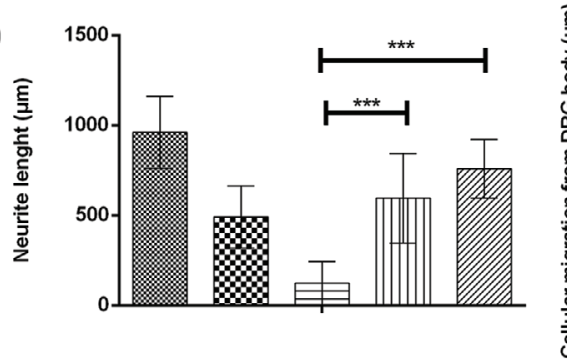

GDNF
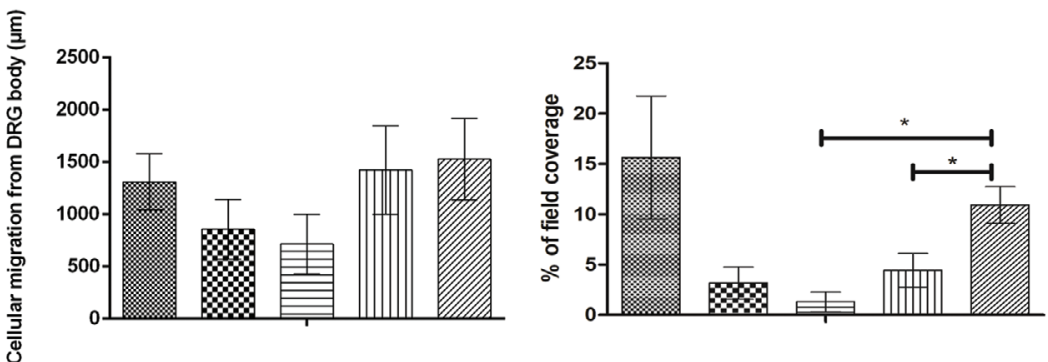

GDNF

GDNF Positive control Negative control $\boxminus$ GDNF/C m $\mathrm{m}$ GDNF/A $\mathrm{A}_{2}$ GDNF/A
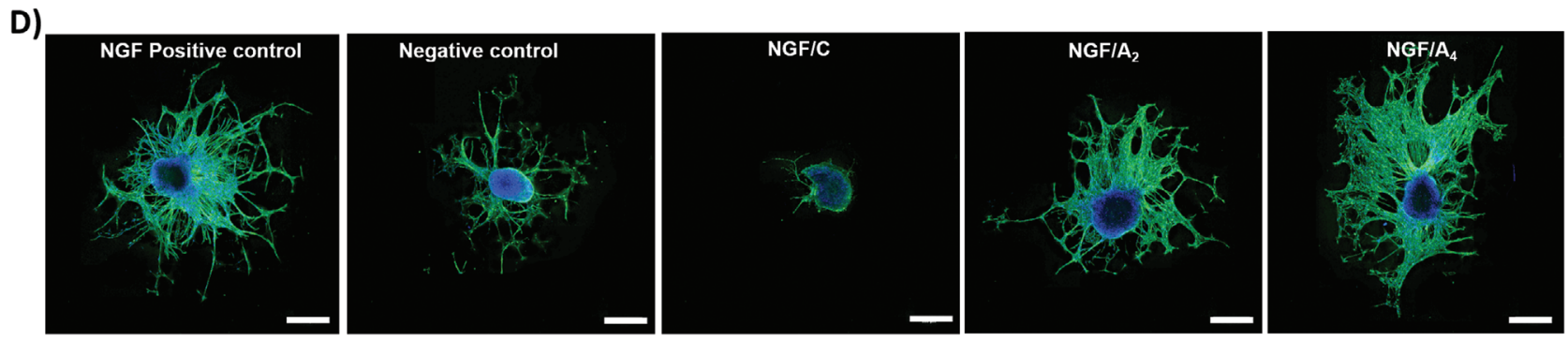

E)

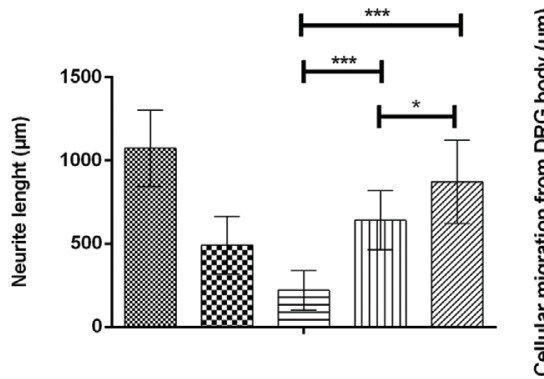

NGF

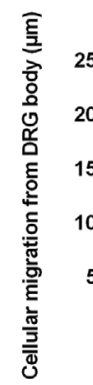

2500
2000
1500
1000
500
0

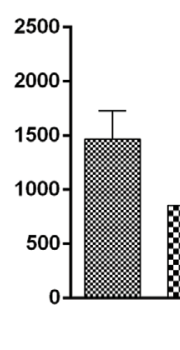

Negative Control

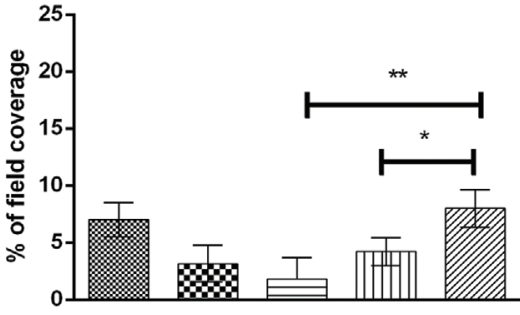

NGF

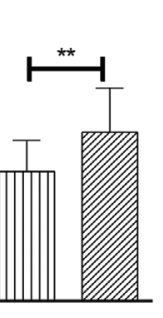

NGFIC
(11) NGF/A

DGF/A 
A)

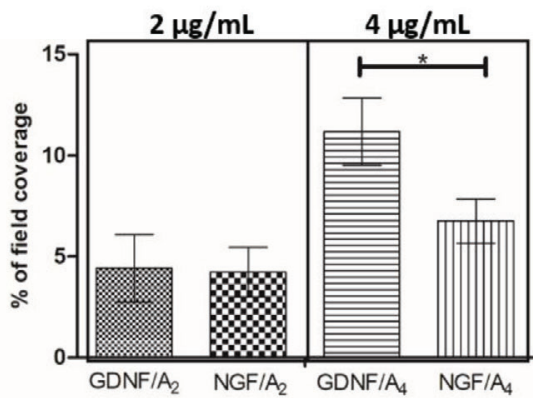

B)
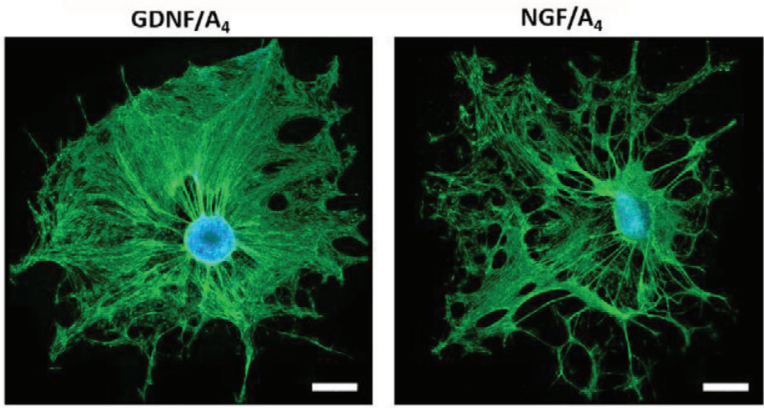

Figure 4. Impact of the different GDNF or NGF formulations on DRGs percentage of field coverage (which reflects both the length and density of neurite outgrowth). When considering the $4 \mu \mathrm{g} \mathrm{mL}^{-1}$ concentration, there is a significant statistical difference between $\mathrm{GDNF} / \mathrm{A}_{4}$ and $\mathrm{NGF} / \mathrm{A}_{4}$ treated $D R G$ in terms of percentage of field coverage. Results were represented as mean $\pm \operatorname{SD}(n=3)$ with at least five DRGs per condition being analyzed in each experiment. Statistically significant differences were represented by $*(p<0.05), * *(p<0.01)$, and $* * * * 0.001)$. Scale bar: $500 \mu \mathrm{m}$.

Table 3. Pinch test results indicating a level of regenerated nerves within the conduit.

\begin{tabular}{lc}
$\begin{array}{l}\text { In vivo implanted } \\
\text { formulations }\end{array}$ & $\begin{array}{c}\text { Number of animals with } \\
\text { positive result to pinch test }\end{array}$ \\
\hline Autograft & $5 / 5(100 \%)$ \\
SF & $1 / 5(20 \%)$ \\
GDNF/A $A_{4}$ & $4 / 5(80 \%)$ \\
\hline
\end{tabular}

of necrosis, acute inflammatory reaction, and calcifications were observed in the implant site and apparently, healthy connective tissue was observed surrounding all the explants.

The results of the pinch test can be seen in the Table 3. At 6 weeks post-surgery, the distal segment of the nerve was isolated and stimulated with a pinch by forceps. Contraction of muscle on the back or retraction of the leg indicates the presence of regenerating fibers in the pinched segment.
After sacrificing the animals, a few standard evaluations were performed, that are capable of indicating the level of success in the nerve regenerative process, by comparing the SF with and without GDNF, against the autograft. Measurements of percentage of muscle weight loss, and quantitative as well as qualitative measurements of longitudinal nerve area in histological images are shown in Figure 5.

In Figure 5A, the different in vivo study groups: autograft, eSF, and GDNF/ $/ \mathrm{A}_{4}$, implanted in the animals are shown. Nerve regeneration was further assessed through the percentage of muscle weight loss, which was determined to be $73.3 \pm 2.7 \%, 79.8 \pm 1.3 \%$ and $76.6 \pm 2.6 \%$ for the autograft, $\mathrm{eSF}$ and GDNF/ $\mathrm{A}_{4}$ groups, respectively, as can be seen in Figure 5B. The results illustrate that plain eSF NGC lead to a significant increase in terms of percentage of muscle weight loss $(p<0.01)$, when compared to the autograft. However, when using GDNF formulation, no significant differences were found. These results suggest that the integration of GDNF decreases the percentage of muscle weight loss, leading to improved functional recovery.

After analyzing the H\&E stained longitudinal sections, a regeneration cable across the $10 \mathrm{~mm}$ nerve gap was observed in $100 \%$ of the autografts $(5 / 5), 80 \%$ in the eSF conduits $(4 / 5)$ and $100 \%$ of the GDNF/A $\mathrm{A}_{4}(5 / 5)$. Furthermore, it was found that the longitudinal sectional nerve area was significantly higher in the GDNF/ $/ \mathrm{A}_{4}$ when compared to eSF (Figure 5C), where the values obtained were $832.68 \pm 293.15,390.55 \pm 130.18$, and, $950.99 \pm$ $239.58 \mathrm{um}^{2}$ for the autograft, eSF conduit and GDNF/ $\mathrm{A}_{4}$, respectively. This result can be seen qualitatively in Figures $5 \mathrm{D}$. The difference in the thickness of the nerve cable can evidently be seen in a cross-section of the NGCs, where the thickness of the cable roughly doubles from eSF to $\mathrm{GDNF} / \mathrm{A}_{4}$ as can be seen in Figure 5E.

Histological procedures can be seen in Figure 6. Explanted samples were cut for histological procedures according to the scheme in Figure 6A.

Semithin cross-sections were observed under a light microscope for a proximal area analysis (PNX section, view at the point of $3 \mathrm{~mm}$ outside the conduit), after osmium tetroxide staining. The number of regenerated myelinated nerve fibers detected (Figure 6B) was similar in the case of the autograft (5443 \pm $985)$ and the GDNF/ $/ A_{4}$ formulation $(4916 \pm 305)$, but significantly higher in the eSF formulation (9281 \pm 2957$)$. The healthy contralateral side contains an average of $6110 \pm 745$ myelinated axons. Qualitatively, the nerve histological image from the GDNF/ $/ \mathrm{A}_{4}$ formulations resembles the one from the autograft, while the eSF formulation presented a distinct microstructure (Figure 6C). Proximal myelinated axons are larger and have a more uniform appearance in the autograft and GDNF/ $\mathrm{A}_{4}$ formulations. This contrasts with the very irregular shaped and sized axons in the eSF formulations. In the same cross-sectional

Figure 3. A) Scheme of the methodology used in the bioactivity assay in vitro. Arrows point to the location of DRGs harvest, close to the spinal cord. B) Representative confocal fluorescent images of DRGs stained with Neurofilament 200 (in green) after being treated with GDNF released from SF conduits. C) Quantification of different parameters related to the DRGs neurite outgrowth, such as neurite length, cellular migration from DRG body and percentage of field coverage after being in contact with GDNF releasing SF conduits. D) Representative confocal fluorescent images of DRGs stained with Neurofilament 200 (in green) after being treated with NGF released from SF conduits. E) Quantification of different parameters related to the DRGs neurite outgrowth, such as neurite length, cellular migration from DRG body and percentage of field coverage after being in contact with NGF releasing SF conduits. Results were represented as mean \pm SD $(n=3)$ with at least five DRGs per condition being analyzed in each experiment. Statistically significant differences were represented by * $(p<0.05)$, 炏 $(p<0.01)$, and $* * * *(p<0.001)$. Scale bar: B,D) $500 \mu \mathrm{m}$. 
A)

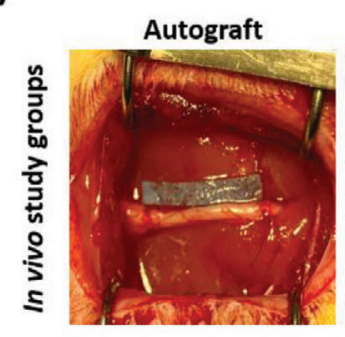

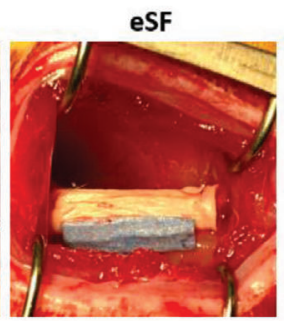

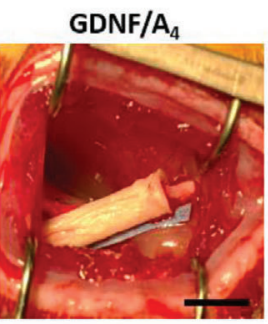

B)

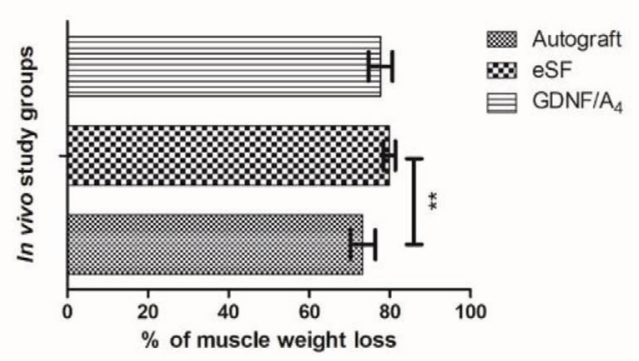

C)

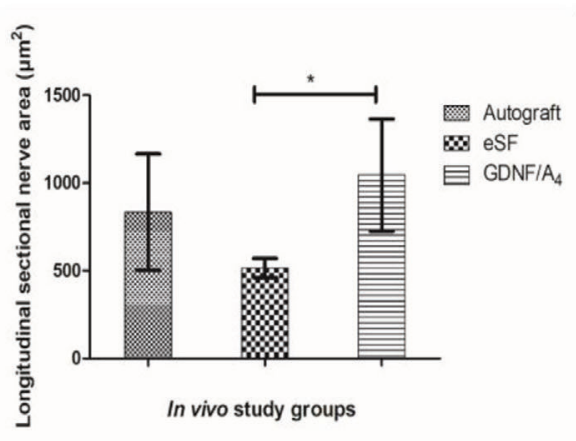

D)

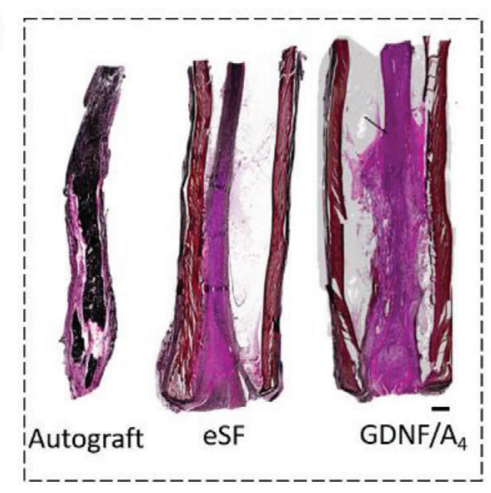

E)

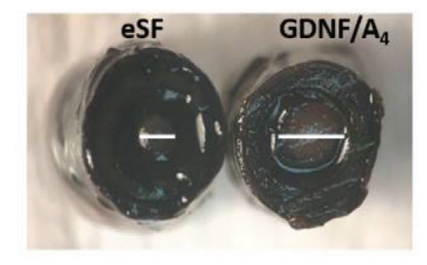

Figure 5. A) Implantation of the different in vivo study groups: autograft, SF and GDNF/A4 $(n=5)$. B) Immediately after animal sacrifice, the percentage of muscle weight loss was determined. C) Quantitative measurements of the longitudinal sectional nerve area. Measurements were made in the middle of the conduit (since the proximal area was cut for histomorphometric analyses, the measurement was performed 7 mm from the distal stump). D) Representative photomicrographs of the longitudinal sections of implanted autograft, SF and GDNF/A4 after hematoxylin-eosin (HE) staining. E) Representative photograph showing the difference in the regenerated nerve cable thickness. Results were represented as mean \pm SD. Statistically significant differences were represented by * $(p<0.05)$, $* *(p<0.01)$, and $* * *(p<0.001)$. Scale bars: A) $500 \mu \mathrm{m} ; \mathrm{D}) 500 \mu \mathrm{m}$; E) $250 \mu \mathrm{m}($ left $)$ and $500 \mu \mathrm{m}$ (right).

area (PNX), blood vessel-like quantification was performed, both qualitatively (Figure S5, Supporting Information) and quantitatively (Figure S6, Supporting Information). The results showed a significant difference between the autograft and the eSF conduits with and without GDNF. A significant increase in the blood vessel-like structure number was verified in the presence of GDNF/A $\mathrm{A}_{4}$ conduits, when compared to nerves reconstructed with plain eSF conduit.

\section{Discussion}

The lack of a pivotal breakthrough in PNR field is leading scientists and medical community to search for new and innovative ways to tackle the problem. Consequently, TE is receiving increasing attention in the treatment of PNI. ${ }^{[27]}$ As the classical FDA-approved NGCs do not provide sufficiently good results in terms of functional recovery, alternative strategies considering the mimicry of natural microenvironment and incorporation of physical and chemical cues for optimal axon guiding became crucial. ${ }^{[28]}$ Furthermore, several works confirmed that NGCs releasing NTFs can improve nerve regeneration across long nerve gaps. ${ }^{[29]}$

Including NTFs in a matrix is a required step since simple administration of NTFs is not sufficient to achieve an effective concentration over a suitable time period that would ultimately promote nerve repair. This is due to their rapid diffusion in the body fluids and destruction by proteolytic enzymes. ${ }^{[30]}$ In this context, the present study demonstrated the beneficial effects of controlled delivery of two PNS prominent growth factors, i.e., GDNF and NGF, in a way to reproduce the native NTF distribution during the nervous system development, therefore stimulating PNR. ${ }^{[18]}$ Moreover, different ways of immobilizing NTFs using a previously described production method of eSF conduits were investigated. ${ }^{[3]}$

SF was selected for developing NGCs because of its wellknown biocompatibility, good mechanical properties and friendly processing. ${ }^{[31]}$ The stability and bioactivity of NTFs during processing is enhanced when using mild processing conditions, which is the case of the eSF, where no organic solvents or harsh reagents were used.

For the crosslinking method, the loading efficiency can be considered as $100 \%$. In contrast, for the absorption method, it has been hypothesized that two different mechanisms contribute to maintaining the NTFs within the eSF conduit. The first relates to the liquid absorption by the porous eSF wall, associated with the swelling capability of the material. The second regards the electrostatic interactions that occur between the negatively charged eSF and the positively charged NTFs, ${ }^{[32]}$ which play a crucial role in this process. In this sense, the affinity of GDNF or NGF proteins to the eSF conduit can partially determine its loading efficiency and give rise to a differential absorption/adsorption. The finest way to calculate the loading efficiency should be using the 


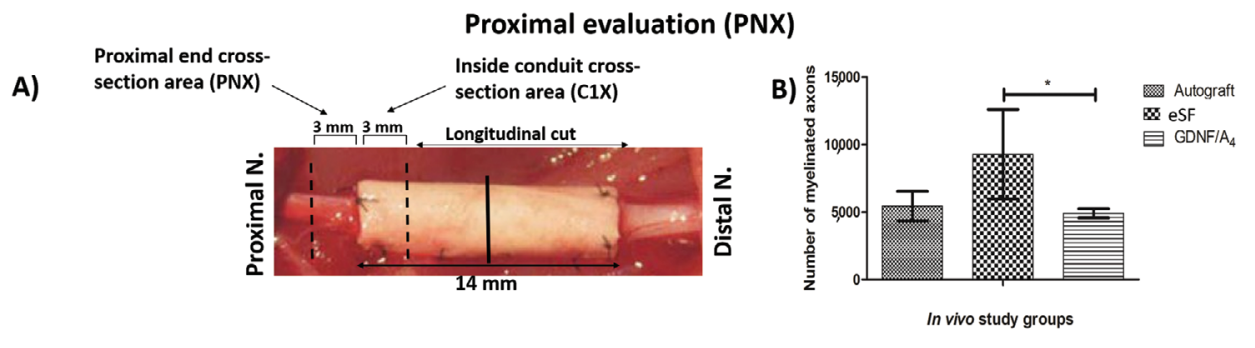

C)

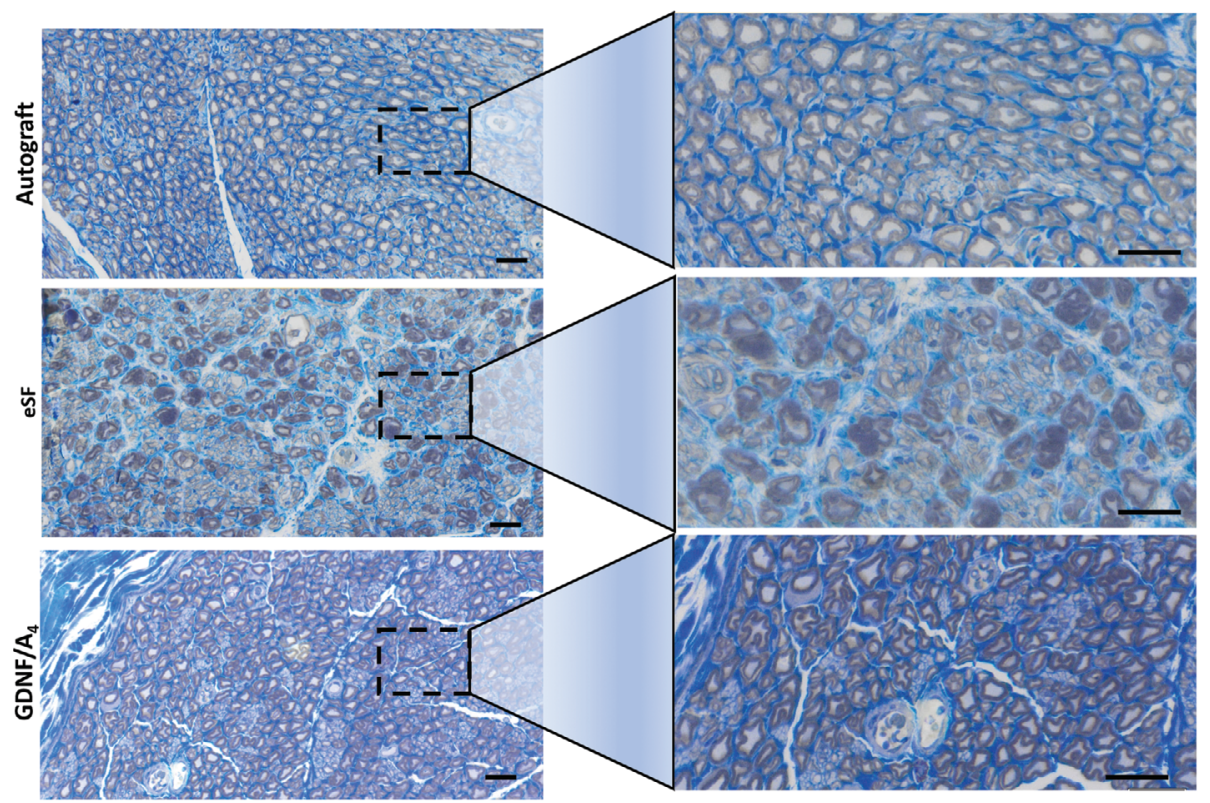

Figure 6. A) Schematic representation of the cross- and longitudinal sections positioning for histological analysis. B) Quantitative evaluation of myelinated axons in the proximal area (PNX) $(n=5)$, where results were represented as mean \pm SD and statistically significant differences were represented by $*(p<0.05), * *(p<0.01)$, and $* * *(p<0.001)$. C) Light microscopy representative images of semithin nerve cross-sections in the PNX cross-sectional area, after osmium tetroxide and toluidine blue staining. On the right, close-up images of each in vivo study groups. Scale bars: $20 \mu \mathrm{m}$.

known concentration to subtract the remaining NTF in the tube. Either way, the ELISA may not be able to interact with degraded NTF. The actual loading efficiency may not be truly reflected by ELISA method. However, the method may be ideal for our study because there is only electrostatic connections or weaker bonds between the NTF and the silk fibroin structure.

It is an evidence that GDNF tends to bind more strongly to the eSF conduit, as seen by the $30 \%$ discrepancy between absorption efficiencies in GDNF and NGF. Without any other variables in the process, this differential absorption could be explained by the molecular/ionic affinity to eSF, which is higher in the case of GDNF, having a higher positive charge when compared to NGF. This is due to the fact that GDNF has an amino-acid rich sequence of $16 \mathrm{~N}$-terminal residues. ${ }^{[33]}$ In this context, it can be predicted that higher NTFs loading implies higher release. The higher affinity binding of GDNF to the SF conduit was visible in its daily release profile, where GDNF has a more sustained release when compared to the burst release observed in the NGF loaded conduits. Similarly, the same principle of higher loading resulting in higher release was verified for both GDNF and $\mathrm{NGF}$, as the GDNF/ $\mathrm{A}_{4}$ and $\mathrm{NGF} / \mathrm{A}_{4}$ formulations released higher amounts of NTFs than GDNF/A $\mathrm{A}_{2}$ and $\mathrm{NGF} / \mathrm{A}_{2}$. When comparing the crosslinking to the absorption method, and given the fact that the same amounts of NTF were loaded in the crosslinked $4 \mu \mathrm{g} \mathrm{mL} \mathrm{m}^{-1}$ and absorption $4 \mu \mathrm{g} \mathrm{mL}^{-1}$ formulations, the minute amounts of NTFs released in the crosslinked formulation evidences the initial hypothesis of stronger molecular entrapment and difficulty to diffuse across the conduit wall. Furthermore, in addition to the expected entrapment of NTFs in the SF matrix, we hypothesize that NTFs could have been covalently linked to each other and to SF tyrosine groups.

It is a fact that SF is a slow-degrading biomaterial. This has been proven in several other publications and the exact degradation profile of the developed enzymatic crosslinked SF conduits has been assessed in another publication. ${ }^{[3]}$ Such degradation profile is obtained by mimicking physiologic conditions (by immersion in Protease XIV at $37^{\circ} \mathrm{C}$ ). Therefore, it is assumed that the degradation profile happening in vivo is similar to what was previously defined. Degradation is a normal process transversal to all biomaterials, and authors expect a constant release of the growth factors as the conduits are being slowly degraded. In fact, the steady and slow degradation of SF when implanted is contributing to the release and consequent action of GDNF in situ.

Overall, the quantity of GDNF and NGF released by GDNF/A 4 and $\mathrm{NGF} / \mathrm{A}_{4}$ formulations, respectively, were similar and in the range of the release values reported in the literature, also 
demonstrating that the absorption/surface binding which occurs during the absorption process allowed the NTFs to maintain a relatively stable and adequate concentration. ${ }^{[18,20]}$ Controlled release of GDNF and NGF in the range of $1-10 \mathrm{ng} \mathrm{mL}^{-1}$ from SF-based NGCs has been suggested to be optimal for nerve repair, ${ }^{[34]}$ which was also verified in our experimental work.

We then investigated the effect of the different formulations on neurite outgrowth of freshly isolated DRGs in vitro, seeded on Matrigel. Matrigel was selected as substrate, since DRGs are highly mechanosensitive and its hydrogel-like stiffness represents the optimal surface for regeneration. ${ }^{[25,35]}$ Furthermore, in the case of negative control, despite the absence of added NTFs, neurite outgrowth was observed since Matrigel can be considered a permissive substrate, ${ }^{[36]}$ very rich in extracellular matrix (ECM) proteins, namely laminin, which is optimal for neuronal cells attachment. ${ }^{[37]}$ Matrigel has been routinely used in preclinical studies for neuronal cells' adhesion and proliferation and its effect in neurite extension has been studied. ${ }^{[37 c]}$ DRG neurons are constituents of the peripheral sensory nervous system and one of the best models to study PNR. ${ }^{[38]}$ The parameters evaluated were the conventional neurite length, cellular migration from DRG body and percentage of field coverage. The pattern of the results was similar for GDNF- and NGF-treated ganglia, where neurite outgrowth reached its maximum in the GDNF/A and $\mathrm{NGF} / \mathrm{A}_{4}$ formulations, followed by the intermediate values obtained by GDNF/A $\mathrm{A}_{2}$ and $\mathrm{NGF} / \mathrm{A}_{2}$. The crosslinked formulations, i.e., GDNF/C and NGF/C, presented the lowest values for neurite outgrowth. Such results are in accordance with their respective releasing profile, where higher amounts of released NTF led to increased neurite length, cellular migration, and percentage of field coverage. The results are also in accordance with other reports, where DRGs were shown to respond to concentrations of $1-2 \mathrm{ng} \mathrm{mL} \mathrm{m}^{-1}$ of GDNF or NGF. ${ }^{[20]}$ Although we initially predicted good outcomes from the crosslinking formulations by preventing NTF burst release, the in vitro results showed otherwise. We can hypothesize that, when compared to the negative control, the poor performance of the crosslinked formulations can be explained by the fact that both NTFs are rich in tyrosine amino acid, possibly leading to an enzymatic crosslinking between SF and NTFs. This covalent binding can result in the slow release of the NTFs, which can be beneficial for long-term applications of the eSF conduits such as the case of the regeneration of longgaps. ${ }^{[39]}$ However, the low amounts released still seem to have an undesired effect on neurite outgrowth, possibly due to binding of the GF to the receptors, occupying or blocking them. In this scenario, the ECM molecules that have a favorable effect in the presence of Matrigel (also present in the negative control) would not be able to act in the crosslinked formulation.

A strong correlation was found between cellular migration and neurite outgrowth. ${ }^{[40]}$ It was evident in the confocal images that cellular components that were isolated with the DRGs, namely Schwann cells and fibroblasts, were paving the way for neurite growth. Only in the case of visible cellular proliferation and spreading, neurite outgrowth would be detected.

Neurite length in the positive control is similar to what is found in the literature, with an average growth of 100-200 $\mu \mathrm{m}$ per day, validating our study. ${ }^{[41]}$ It has been shown that Schwann cell proliferation and migration play a crucial role during nerve regeneration. ${ }^{[2]}$ Therefore, it is possible to predict that which
NTF is able to promote a higher Schwann cell migration, is also capable of further enhancing the in vivo outcomes. Through confocal images, it was possible to observe that the path of neurites outgrowth was guided by cellular components (fibroblasts and Schwann cells). Therefore, the two parameters are connected as demonstrated by the fact that the greater cellular migration led to more extensive the neurite growth. It was interesting to be able to correlate those two facts, that were true for all formulations (please see Figure S3 in the Supporting Information). In conclusion, to have neurite elongation, cellular migration is also necessary and both parameters are intrinsically correlated. It was observed that less amounts of released NGF (up to $5 \mathrm{~d}$ ) are more efficient and have a more intense effects on neurite elongation as compared to GDNF (since less ng released provide similar neurite extension). But when comparing NGF/A2 and NGF/A4, the higher amount of NGF released by NGF/A4 is crucial and makes the significant difference. If this were the only focus of the study, NGF would be the first choice. However, neurite density is an important parameter as well, and at this point the clear front-runner is GDNF. Concerning DRG in vitro study, DRGs are considered sensory neurons and they appear responds to NGF in a different manner. Also, from the study we can tell NGF is more dose depend than GDNF.

Together with the ELISA results, the in vitro biological tests allowed to exclude any crosslinked formulation for further studies and elect the GDNF/ $/ \mathrm{A}_{4}$ and $\mathrm{NGF} / \mathrm{A}_{4}$ as the most promising formulations. In our study, GDNF and NGF prompted similar neurite elongation, but GDNF increased the neurite density. In this context, GDNF/A $\mathrm{A}_{4}$ was selected for the in vivo evaluation since its percentage of field coverage was significantly higher than NGF/A 4 .

Considering the results from the in vivo study, the presence of GDNF significantly promoted motor reinnervation and motor fibers survival, as the muscle weight loss or atrophy was significantly avoided in the gastrocnemius muscle, when compared to the SF samples, which was critical for functional recovery. ${ }^{[43]}$ On the other hand, in relation to the pinch test, the reflex shown by most of the animals in the GDNF/A $\mathrm{A}_{4}$ study group, proves that GDNF could act on sensory reinnervation. ${ }^{[4]}$ According to our literature research, the pinch test is "a test where reflex movement of the back muscles or retraction of the leg indicate the presence of sensory fibers in the pinched segment while no response indicates as the absence of such fibers." ${ }^{[44]}$ Such test can be done by pinching the nerve segment distal to the conduit, as was performed in our experimental work. These findings could be related to the in vitro biological observations, where sensory DRGs positively respond to GDNF.

Longitudinal H\&E sections allowed to study the general nerve regeneration potential of the different formulations. A complete fibrin nerve cable formation is essential for obtaining a complete nerve regeneration with functional recovery. Longitudinal sectional nerve area and number of samples in which the nerve cable was able to cross the $10 \mathrm{~mm}$ gap, point to GDNF supplemented SF conduits to significantly outperform the SF formulation.

It has to be taken in consideration that the active retrograde transport of growth factors is crucial for the normal function of nerves, and disorders in this mechanism result in neurodegeneration, neuropathies and cell death. ${ }^{[45]}$ Signaling proteins frequently travel from the axon terminals to the cell body in order to 
trigger and stimulate their signaling pathways. In the case of an injury, NTFs released by the native tissue are transported to the cell bodies, in order to promote survival mechanisms. ${ }^{[46]}$ In this experimental work, a simple osmium tetroxide/toluidine blue staining allowed to assess that GDNF/A $\mathrm{A}_{4}$ formulation showed better retrograde nerve protection and nerve trunk protection when compared to the SF conduit. ${ }^{[47]}$ In the proximal area, without the GDNF being transported backwards, SF reconstructed nerves suffered from significant degeneration. It is noteworthy that many axons began sprouting from proximal site. However, this indicated that nerve was severely damaged. ${ }^{[48]}$ In this scenario, the regeneration may be delayed or failed.

Only recently the scientific community has realized the importance of vascularization in PNR. In fact, the lack of blood supply is one of the most important and limiting factors impairing regeneration. ${ }^{[49]}$ Although no specific vascularization staining was performed, blood vessels-like structures are well identified in the toluidine blue staining because of the visible endothelial wall, shape and presence of erythrocytes. ${ }^{[50]}$ The significant increment on blood vessel-like structure number in the nerve regenerated within the GDNF/A $\mathrm{A}_{4}$ conduit demonstrated the beneficial environment provided by the developed NGC, while confirming the direct relationship between nerve regeneration and angiogenesis. ${ }^{[51]}$ It has been shown that not only the new vasculature reduces hypoxia in the regenerating nerve, but Schwann cells use the blood vessels present in the proximal nerve as "tracks" to cross and migrate through the bridge, taking regrowing axons with them. ${ }^{[52]}$ These results confirm other reports, where certain GDNF peptides are described as potent angiogenic factors. ${ }^{[53]}$ Thus, all evidence withdrew from this study points to the gainful effects of trophically functionalizing NGCs.

The advantageous effects of an NGC on nerve repair will difficultly achieve a similar performance than an autograft. Still, the developed GDNF loaded SF conduit performed similarly to the autograft in the variety of parameters evaluated, namely in what concerns the quality of the regenerated nerve cable and recovery of nerve function (assessed by the pinch test). Although a 6-week period for nerve regeneration can be considered short and early for some assessments, such as electrophysiological evaluations, the fact that significant statistical differences were found between the studied formulations in some parameters, confirms that effective conclusions can be made from this study.

\section{Conclusion}

In this study, we have demonstrated that the enzymatically crosslinked silk fibroin conduits may be loaded with two different neurotrophic factors, via different methodologies. They presented distinctive releasing profiles, according to their affinity to silk fibroin. In vitro, the superior releasing profile of GDNF could be assessed when it was incorporated by the adsorption method at the concentration of $4 \mu \mathrm{g} \mathrm{mL}{ }^{-1}$. These results were confirmed when DRGs' neurite density was significantly enhanced in the presence of such formulation. Furthermore, the valuable effects of GDNF/A $\mathrm{A}_{4}$ on improving nerve regeneration in vivo resulted in a suitable environment for peripheral nerve regeneration. Implanting such formulation on a $10 \mathrm{~mm}$ rat sciatic nerve defect acted on both sensory and motor fibers, as was perceived by the specific assays of pinch test and percentage of muscle weight loss. Related to the histological and histomorphometrical evaluation, when using the absorbed GDNF formulation, proximal nerve protection was found to be more robust when compared to plain silk fibroin conduit and comparable to the autograft, via retrograde transport of GDNF. Vascularization was also significantly increased by the addition of GDNF to silk fibroin, which represents another gain in terms of nerve regeneration.

Overall, the addition of GDNF to the silk fibroin conduit could be one possible treatment to prevent neurodegeneration. The findings from this experimental work could significantly contribute to TE field. It is our firm belief that the developed GDNFincorporating silk fibroin conduit is a promising candidate for future studies, where cellular therapies and guiding luminal fillers might be included, creating new and multifactorial therapeutic alternatives for the treatment of peripheral nerve injuries.

\section{Experimental Section}

Materials: Bombyx mori cocoons were supplied by the Portuguese Association of Parents and Friends of Mentally Disabled Citizens (APPACDM, Portugal). Hydrogen peroxide was purchased from Panreac (Spain). Human recombinant GDNF (cat\#450-10-10 $\mu \mathrm{g}$ ) and Human recombinant NGF (cat\#450-01-100 $\mu \mathrm{g}$ ) were purchased from Peprotech (USA). Human GDNF Duoset ELISA kit (cat\#DY212) and Human $\beta$-NGF Duoset ELISA kit (cat\#DY256) were purchased from R\&D Systems (USA). Corning Matrigel phenol red-free was purchased from VWR (USA). Other materials and reagents were purchased from Sigma-Aldrich (USA).

Preparation of Silk Fibroin (SF) Conduits: The purification of SF and consequent preparation of eSF NGCs was prepared as described elsewhere. ${ }^{[3]}$ Briefly, SF was detached from the other main protein present in the cocoons, sericin. For this purpose, the cocoons were immersed in a $0.02 \mathrm{M}$ boiling sodium carbonate solution for $1 \mathrm{~h}$, followed by rinsing with abundant distilled water. The obtained SF was then dissolved in a $9.3 \mathrm{M}$ lithium bromide solution at $70^{\circ} \mathrm{C}$ for $1 \mathrm{~h}$ and dialyzed (benzoylated dialysis tubing, MWCO: $2 \mathrm{kDa}$ ) against distilled water for $48 \mathrm{~h}$ to remove contaminations. The purified SF was concentrated against a $20 \mathrm{wt} \%$ poly (ethylene glycol) solution for at least $6 \mathrm{~h}$, and the final concentration was determined by weighting the dry product after drying at $70{ }^{\circ} \mathrm{C}$ overnight. After determining the concentration, the SF solution was diluted to $16 \mathrm{wt} \%$ with distilled water, and mixed with horseradish peroxidase solution (HRP type VI, $\left.0.84 \mathrm{mg} \mathrm{mL}^{-1}, 100 \mu \mathrm{L}\right)$ and hydrogen peroxide solution $\left(\mathrm{H}_{2} \mathrm{O}_{2}, 0.36 \mathrm{wt} \%\right.$, $65 \mu \mathrm{L}$ ) for enzymatic crosslinking following the procedure described by Yan et al. ${ }^{[54]}$ In order to produce the conduits, the previous mixture was injected within the space between two concentric cylinder molds, where the outer cylinder had a diameter of $4 \mathrm{~mm}$ and the inner cylinder had a diameter of $2 \mathrm{~mm}$. The system was incubated at $37^{\circ} \mathrm{C}$ for the period of $1 \mathrm{~h}$ in order to induce gelation. After gelation, a quick immersion in liquid nitrogen is needed to remove the outer mold, followed by an immersion in ethanol 100 vol\% to induce permanent crystalline $\beta$-sheet conformation and to remove the inner mold. The obtained hollow conduits were subjected to a process of lyophilization for $72 \mathrm{~h} .{ }^{[3]}$ Final conduits had a wall thickness of $\approx 700 \mu \mathrm{m}$, a lumen diameter of $\approx 5 \mathrm{~mm}$ and an external pore size in the range of 3-6 $\mu \mathrm{m}$. Conduits were cut to a total length of $14 \mathrm{~mm}$ for all assays.

Physical Characterization of the Conduits: The morphology of the produced eSF conduits was assessed under a scanning electron microscope (model S360, Leica, Cambridge, UK). The specimens were sputter coated with gold, prior to the analysis of surface morphology and microstructure.

Incorporation of NTFs: The incorporation of NTFs was achieved via two different methods, which will be designated throughout the manuscript: i) crosslinking method, and ii) absorption method. For the crosslinking method, a NTF solution of $4 \mu \mathrm{g} \mathrm{mL}^{-1}$ in PBS was added to $1 \mathrm{~mL}$ of aqueous $\mathrm{SF}$ solution. As described in the preparation of the conduits, the solution was mixed with $\mathrm{HRP}$ and $\mathrm{H}_{2} \mathrm{O}_{2}$, allowing the NTFs to be entrapped in 
the conduits' walls after crosslinking. The processing was then carried out as previously described. For the adsorption method, the conduits' preparation was conducted following the indicated protocol. Final lyophilized conduits (made of $1 \mathrm{~mL}$ of SF solution) were immersed in $1 \mathrm{~mL}$ of a $4 \mu \mathrm{g}$ $\mathrm{mL}^{-1}$ or $2 \mu \mathrm{g} \mathrm{mL}-1$ of NTF solution in PBS for $3 \mathrm{~h}$ at $37^{\circ} \mathrm{C}$. A second step of lyophilization was performed. The NTFs incorporated in the eSF conduits by crosslinking and absorption were GDNF and NGF. A table identifying the six produced samples is shown in Table 1.

Determination of NTFs' Loading Efficiency in eSF Conduits: After the production of the different samples indicated in Table 1, the NTFs' loading efficiency in the eSF conduits was determined using the enzyme-linked immunosorbent assay (ELISA) kits for GDNF and NGF, respectively. These experiments were only carried for the absorption method, since it can be predicted that $100 \%$ of NTF was incorporated in the crosslinking method. Considering that the initial NTF solutions had the concentration of 2 and $4 \mu \mathrm{g} \mathrm{mL}^{-1}$, the NTF solutions that remained after the absorption process were frozen at $-20^{\circ} \mathrm{C}$, stored and later subjected to ELISA quantification. In order to determine the percentage of NTFs' loading efficiency, Equation (1) was applied, where $\mathrm{Ci}$ represents the initial NTF concentration in which the conduit was soaked, and $x$ represents NTF quantification obtained by ELISA. A triplicate of samples was measured in three independent experiments, and the results were expressed as mean $\pm S D$.

$\%$ NTF of loading efficiency $=100-\frac{x \times 100}{C i}$

In Vitro Release of NTFs from the Nerve Conduits: The amount of NTFs released from the eSF conduits was quantified by ELISA according to the manufacturer's instructions. Each eSF conduit, with the size of $14 \mathrm{~mm}$ in length, was incubated in $1 \mathrm{~mL}$ of release medium (1 wt\% BSA in PBS) at $37^{\circ} \mathrm{C}$, with shaking at $60 \mathrm{rpm}$, for $50 \mathrm{~d}$. The supernatant was collected and substituted with $1 \mathrm{~mL}$ of fresh release medium every $24 \mathrm{~h}$. The absorbance was measured in the collected solutions at $450 \mathrm{~nm}$ using a plate reader (Tecan, Spark 10M, Switzerland). The NTFs concentration was determined by means of comparing the reading to the obtained standard curve after normalization

In Vitro Isolation and Culturing of DRGs for NTFs Bioactivity Assay: The biological activity of the NTFs released from the eSF conduits was assessed by determining the axonal outgrowth of DRGs. DRGs explants were isolated from neonatal rat pups (considered neonatal from day 1 to day 5). They were aseptically harvested and cleaned with dissecting forceps to remove the tails and excessive connective tissue. After cutting the DRGs in halves, they were immediately seeded on Matrigel coated wells. The Matrigel coating was done at least $3 \mathrm{~h}$ before the DRGs seeding, using cold Matrigel $\left(4^{\circ} \mathrm{C}\right)$ and allowing it to polymerize at $37^{\circ} \mathrm{C}$. The DRGs were kept in regular Dulbecco's modified Eagle medium (DMEM) supplemented with 10 vol\% fetal bovine serum and 1 vol\% penicillin/streptomycin for the first $24 \mathrm{~h}$, allowing cellular attachment. DRGs were plated at a density of two DRGs per well. At the same time, the NTF loaded eSF conduits were immersed in $1 \mathrm{~mL}$ of complete DMEM and incubated at 37 ${ }^{\circ} \mathrm{C}$ and $60 \mathrm{rpm}$, producing a NTF enriched media, denominated as conditioned media. Every $24 \mathrm{~h}$, the conditioned media was transferred to the wells containing DRGs and replaced with fresh media. For this assay, the negative control consisted of DRGs treated with regular DMEM and the positive control comprised DMEM media supplemented with $50 \mathrm{ng} \mathrm{mL}^{-1}$ of GDNF or NGF. DRGs were cultured with this methodology for $5 \mathrm{~d}$, at 37 ${ }^{\circ} \mathrm{C}$ and $5 \% \mathrm{CO}_{2}$. This assay was performed three independent times, with six DRGs per condition each time.

Immunocytochemistry: After $5 \mathrm{~d}$ of incubation, DRGs and cells were fixed with 4 vol\% paraformaldehyde for $30 \mathrm{~min}$. DRGs were washed and treated with Triton 0.2 vol\% in PBS for 10 min for permeabilization purposes. The next step consisted in adding blocking buffer comprising $3 \mathrm{wt} \%$ BSA in PBS, at room temperature (RT), for $30 \mathrm{~min}$. The primary antibody, diluted in $1 \mathrm{wt} \%$ BSA in PBS, was then added, and the mixture incubated for $1 \mathrm{~h}$ at RT (1:150, Monoclonal Anti-Neurofilament 200, produced in mouse). After washing with PBS, the secondary antibody was immediately added and incubated for $1 \mathrm{~h}$ at RT, protected from light (1:500, Alexa Fluor
488 goat anti-mouse). The last step consisted in nuclei staining, in which Hoechst staining was applied for 5 min (1:5000).

Image Acquisition and Data Analysis: The stained DRGs were analyzed using the confocal laser microscope (Leica). Confocal images were acquired and mounted together to fit in a black square (with the exact same size in all images) so that it would be possible to do image analysis and comparable quantifications. Fluorescent images were imported to Image| software (Fiji) and three parameters were analyzed and quantified concerning the DRGs neurite outgrowth: neurite length, cellular migration, and percentage of field coverage. ${ }^{[55]}$ For neurite length and cellular migration quantifications, the average of the 20 longest neurites and the 20 furthest cells in each image were measured, in a total of five images per condition. ${ }^{[56]}$ For percentage of field coverage, Image) was also used to convert the pictures to black and white (threshold of 58 for all images) and the $\%$ of white pixels in the black field was quantified. After statistical analysis, the group presenting the best performance was selected for the animal study and compared with autograft.

Animal Study: Three different study groups were investigated in vivo on a $10 \mathrm{~mm}$ sciatic nerve defect in rats, including: autograft $(n=5)$, eSF $(n=5)$ and GDNF/A $4(n=5)$. The autograft group was the positive control and the eSF group was the negative control to assess the efficacy of GDNF/A $/ A_{4}$. The degree of nerve regeneration was assessed after 6 weeks post-implantation. All animals were carefully maintained according to methods approved by the Institutional Animal Care and Use Committee at the Rutgers University and the National Institutes of Health regulations and standards for animal usage were followed.

Surgical Procedure: Male Lewis rats weighing 250-300 g (Charles River Labs, USA) were anesthetized with isoflurane inhalation (1-5\% isoflurane in $100 \% \mathrm{O}_{2}$ ) via an appropriate face mask, which was kept throughout the surgical procedure for anesthesia maintenance. Animals were kept on a heated pad until they recovered from anesthesia. Before the surgery, Buprenorphine (for analgesic purposes, $0.05 \mathrm{mg} \mathrm{kg}^{-1}$ ) and Baytril (for antibiotic purposes, $5 \mathrm{mg} \mathrm{kg}^{-1}$ ) were administered subcutaneously. After shaving the surgical site on the left limb, local anesthesia (Bupivacaine, diluted to $0.25 \%, 2.5 \mathrm{mg} \mathrm{kg}^{-1}$ ) was also administered in the place of incision. The shaved area was then cleaned by alternating applications of chlorhexidine and 70 vol\% isopropyl alcohol in circular motions.

Nerve cutting tools were printed from poly(lactic acid) on a Monoprice fused deposition modeling printer. Tools were designed with a hook presenting an inner diameter of $2.5 \mathrm{~mm}$ and a scalpel alignment groove $700 \mu \mathrm{m}$ in width. Tools had a beveled tip and specific dimensions to allow for minimal tissue damage while exposing and sectioning the nerve. Printed tools were cleaned in ethanol, individually packed and surgically sterilized with ethylene oxide. A scheme of a nerve cutting tool can be seen in Figure S6 (Supporting Information).

For the conduits' groups, a $5 \mathrm{~mm}$ section of the sciatic nerve was removed with the aid of the above-described nerve cutting tool, and the nerve stumps were allowed to retract to form a $10 \mathrm{~mm}$ gap. Sterile conduits (14 $\mathrm{mm}$ in length) were first hydrated for $10 \mathrm{~min}$ with sterile saline and then sutured to the nerve stumps using two 9-0 epineurial sutures on each end, maintaining the $10 \mathrm{~mm}$ gap between the stumps. In that process, nerve stumps were secured approximately $1 \mathrm{~mm}$ into each end of the conduit. In the case of autografts, a $10 \mathrm{~mm}$ segment of nerve was removed, reversed, and sutured back in the gap using 9-0 sutures on each end. Both muscular and skin tissues were sutured in layers using absorbable 5-0 sutures. For postoperative analgesia, buprenorphine SR $\left(1 \mathrm{mg} \mathrm{Kg}^{-1}\right)$ was administered $8 \mathrm{~h}$ after the surgery. Animals were checked at least once a day.

Six weeks after surgery, rats were deeply anesthetized using the same method. The analyzed groups were harvested (SF conduits containing the regenerated nerves and the autografts were collected, on the experimental side), as well as a segment of uninjured nerve on the contralateral side, for comparison purposes. Still under general anesthesia, animals were euthanized by carbon dioxide asphyxiation.

Pinch Test: A pinch test was performed in a way to assess sensory functional recovery. The distal nerve segment was pinched with a pair of forceps to determine any reflex of the ipsilateral side. Every animal of every group was subjected to this test, repeated at least three times. ${ }^{[44,57]}$ 
Gastrocnemius Muscle Harvest and Weight Ratio: Upon nerve harvest and after animal sacrifice, the gastrocnemius muscle of both hind limbs (operated side and contralateral healthy side) were immediately harvested by exposing the musculature via a knee to ankle longitudinal skin incision. The muscles were harvested from origin to insertion and weighed with an electronic balance in order to be able to determine the muscle atrophy resultant from muscle denervation. Results were expressed as the percentage of the left side (reconstructed with eSF conduits) to the nonoperated contralateral side, as a mean of five samples per condition (mean \pm SD).

Histological Preparation: Immediately after harvesting, both reconstructed nerves and healthy contralateral nerves were subjected to in situ fixation by immersing in 4 vol\% paraformaldehyde. Sections $3 \mathrm{~mm}$ into the NGC (after CIX area) were cut longitudinally and further processed for hematoxylin and eosin (H\&E) staining. Briefly, samples were deparaffinized in xylol, hydrated through decreasing ethanol series (100\%, $96 \%$, $70 \%$, and 50 vol\% alcohol) and finally washed in distilled water. After immersion in hematoxylin, samples were rinsed in running soft tap water. Samples were then immersed in an $80 \%$ alcohol solution and briefly in the eosin staining. Slides were dehydrated through 96 vol\% ethanol, absolute ethanol and finally cleared in xylol and mounted. Longitudinal sections were analyzed for longitudinal nerve area and assessment of regenerated nerve cable formation.

Histomorphometric Analysis of Explanted Nerve: Immediately after harvesting, both reconstructed nerves and healthy contralateral nerves were subjected to in situ fixation by immersing in 4 vol\% paraformaldehyde. The nerve was then harvested and processed as previously described. [58] Briefly, the explant was embedded in epoxy resin and $1 \mu \mathrm{m}$ thick sections were obtained and stained with osmium tetroxide followed by 1 vol\% toluidine blue. Cross-sections of the proximal nerve $(3 \mathrm{~mm}$ from the conduit, PNX) were analyzed for axon count. Image analysis was performed using Image) software.

Statistical Analysis: Numerical data were represented as mean \pm SD. At least three specimens were used in each condition. Statistical analysis was performed using the GraphPad Prism 5.0 (GraphPad Software, USA) For ELISA quantification analysis, the experiments were repeated three independent times $(n=3)$ and represented as mean \pm SD. A ShapiroWilk test was used to ascertain about the data normality. Subsequently, a Kruskal-Wallis test was performed followed by Dunn's Multiple comparison test, where the significance level was set to $p<0.05$. The biological in vitro experiments were repeated three independent times $(n=3)$ and represented as mean \pm SD. For in vitro statistical analyses, a twotailed Mann Whitney test was used $(p<0.05)$. For the in vivo assay, 15 rats were used, with five specimens for each condition. For in vivo image analysis, the values represent mean \pm SD. A two-tailed Mann Whitney test was used $(p<0.05)$. For percentage of muscle weight loss, results were expressed as the percentage of the left side (reconstructed with eSF conduits) to the nonoperated contralateral side, as a mean of five samples per condition. Subsequently, a Kruskal-Wallis test was performed followed by Dunn's Multiple comparison test, where the significance level was set to $p<0.05$.

\section{Supporting Information}

Supporting Information is available from the Wiley Online Library or from the author.

\section{Acknowledgements}

This work was supported by C.R.C.'s Ph.D. scholarship (Norte-08-5369FSE-000037) and awarded FLAD scholarship (Proj. 29/2018). The FCT distinctions attributed to J.M.O. (IF/00423/2012 and IF/01285/2015) and J.S.-C. (IF/00115/2015) under the Investigador FCT program are also greatly acknowledged. The authors also thank ljaz Ahmed from the BME department and Joe Steel from NJCBM, both from Rutgers University, for their important contribution in this scientific paper. The New Jersey Center for Biomaterials at Rutgers University provided additional support for this study. The authors acknowledge the project: "Nano-accelerated nerve regeneration and optogenetic empowering of neuromuscular functionality" (ref. PTDC/NAN-MAT/29936/2017).

\section{Conflict of Interest}

The authors declare no conflict of interest.

\section{Keywords}

nerve guidance conduits, neurotrophic factors, peripheral nerve regeneration, retrograde transport, silk fibroin

Received: May 4, 2020

Revised: October 15, 2020

Published online:

[1] M. Wiberg, G. Terenghi, Surg. Technol. Int. 2003, 11, 303.

[2] D. Grinsell, C. P. Keating, Biomed. Res. Int. 2014, 2014, 1.

[3] C. R. Carvalho, J. B. Costa, A. da Silva Morais, R. López-Cebral, J. Silva-Correia, R. L. Reis, J. M. Oliveira, Adv. Healthcare Mater. 2018, 7, 1800186

[4] R. Deumens, A. Bozkurt, M. F. Meek, M. A. Marcus, E. A. Joosten, J. Weis, G. A. Brook, Prog. Neurobiol. 2010, 92, 245.

[5] N. Patel, K. Lyon, J. Huang, Neural Regener. Res. 2018, 13, 764.

[6] A. Magaz, A. Faroni, J. E. Gough, A. J. Reid, X. Li, J. J. Blaker, Adv. Healthcare Mater. 2018, 7, 1800308.

[7] a) S. Das, U. Bora, B. B. Borthakur, in Silk Biomaterials for Tissue Engineering and Regenerative Medicine (Ed: S. C. Kundu), Woodhead Publishing, Cambridge 2014, p. 41; b) D. Jao, X. Mou, X. Hu, J. Funct. Biomater. 2016, 7, 22.

[8] P. J. Horner, F. H. Gage, Nature 2000, 407, 963.

[9] P. J. Kingham, G. Terenghi, J. Anat. 2006, 209, 511.

[10] K. Tajdaran, K. Chan, T. Gordon, G. H. Borschel, Exp. Neurol. 2019, 319, 112817.

[11] M. F. Meek, J. H. Coert, Ann. Plast. Surg. 2008, 60, 110.

[12] M. Blais, P. Lévesque, S. Bellenfant, F. Berthod, Tissue Eng., Part A 2013, 19, 1655.

[13] K. M. Rich, J. R. Luszczynski, P. A. Osborne, E. M. Johnson Jr, J. Neurocytol. 1987, 16, 261.

[14] V. Wong, R. Arriaga, N. Y. Ip, R. M. Lindsay, Eur. J. Neurosci. 1993, 5, 466.

[15] A. Blesch, M. H. Tuszynski, J. Comp. Neurol. 2003, 467, 403.

[16] J. C. Petruska, L. M. Mendell, in Encyclopedia of Neuroscience (Ed: L. R. Squire), Academic Press, Oxford 2009, p. 71

[17] P. R. Ho, G. M. Coan, E. T. Cheng, C. Niell, D. M. Tarn, H. Zhou, D. Sierra, D. J. Terris, Arch. Otolaryngol., Head Neck Surg. 1998, 124, 761.

[18] S. Tang, J. Zhu, Y. Xu, A. P. Xiang, M. H. Jiang, D. Quan, Biomaterials 2013, 34, 7086 .

[19] S. Wang, Q. Cai, J. Hou, J. Bei, T. Zhang, J. Yang, Y. Wan, J. Biomed. Mater. Res., Part A 2003, 66A, 522.

[20] S. Madduri, M. Papaloïzos, B. Gander, Biomaterials 2010, 31, 2323.

[21] L. Uebersax, M. Mattotti, M. Papaloïzos, H. P. Merkle, B. Gander, L. Meinel, Biomaterials 2007, 28, 4449.

[22] a) P. D. Chowdary, D. L. Che, K. Zhang, B. Cui, Biophys. J. 2015, 108, 2691; b) K. Ito, H. Enomoto, J. Biochem. 2016, 160, 77.

[23] N. Abe, V. Cavalli, Curr. Opin. Neurobiol. 2008, 18, 276.

[24] U. Paravicini, K. Stoeckel, H. Thoenen, Brain Res. 1975, 84, 279.

[25] G. Rosso, P. Young, V. Shahin, Cell. Physiol. Biochem. 2017, 44, 1263.

[26] G. Corfas, M. O. Velardez, C.-P. Ko, N. Ratner, E. Peles, J. Neurosci. 2004, 24, 9250 
[27] F. Liu, H. Lin, C. Zhang, Methods Mol. Biol. 2016, 1453, 33.

[28] C. Liu, C. Wang, Q. Zhao, X. Li, F. Xu, X. Yao, M. Wang, Biomed. Mater. 2018, 13, 044107

[29] N. Xiao, Q.-T. Le, Arch. Immunol. Ther. Exp. 2016, 64, 89.

[30] B. Wang, J. Yuan, J. Xu, X. Chen, X. Ying, P. Dong, Exp. Ther. Med. 2017, 13, 178

[31] R. D. Abbott, E. P. Kimmerling, D. M. Cairns, D. L. Kaplan, ACS Appl. Mater. Interfaces 2016, 8, 21861.

[32] S. Madduri, B. Gander, J. Peripher. Nerv. Syst. 2010, 15, 93.

[33] I. Alfano, P. Vora, R. S. Mummery, B. Mulloy, C. C. Rider, Biochem. J. 2007, 404, 131.

[34] S. Catrina, B. Gander, S. Madduri, Eur. J. Pharm. Biopharm. 2013, 85, 139.

[35] G. Rosso, I. Liashkovich, P. Young, D. Rohr, V. Shahin, Nanomed.: Nanotechnol., Biol., Med. 2017, 13, 493.

[36] L. N. Novikova, A. Mosahebi, M. Wiberg, G. Terenghi, J. O. Kellerth, L. N. Novikov, J. Biomed. Mater. Res., Part A 2006, 77A, 242.

[37] a) C. S. Hughes, L. M. Postovit, G. A. Lajoie, Proteomics 2010, 10, 1886; b) S. J. Lee, W. Zhu, L. Heyburn, M. Nowicki, B. Harris, L. G. Zhang, IEEE Trans. Biomed. Eng. 2017, 64, 408; c) S. Wu, M. S. Chen, P. Maurel, Y. S. Lee, M. B. Bunge, T. L. Arinzeh, J. Neural Eng. 2018, 15, 056010.

[38] Saijilafu, F.-Q. Zhou, J. Vis. Exp. 2012, 66, 4141.

[39] a) C. Xue, H. Zhu, D. Tan, H. Ren, X. Gu, Y. Zhao, P. Zhang, Z. Sun, Y. Yang, J. Gu, Y. Gu, X. Gu, J. Tissue Eng. Regener. Med. 2018, 12, e1143; b) Y. C. Lin, M. Ramadan, M. Hronik-Tupaj, D. L. Kaplan, B. J. Philips, W. Sivak, J. P. Rubin, K. G. Marra, Ann. Plast. Surg. 2011, 67, 147.

[40] a) D. M. Thompson, H. M. Buettner, Ann. Biomed. Eng. 2006, 34, 161; b) R. P. Bunge, J. Exp. Biol. 1987, 132, 21.

[41] D. A. Tonge, J. P. Golding, M. Edbladh, M. Kroon, P. E. Ekstrom, A. Edstrom, Exp. Neurol. 1997, 146, 81.

[42] K. R. Jessen, R. Mirsky, Front. Cell. Neurosci. 2019, 13, 33.
[43] N. Mirzakhani, A. A. Farshid, E. Tamaddonfard, M. Imani, A. Erfanparast, F. Noroozinia, Life Sci. 2018, 215, 22.

[44] G. Lundborg, L. Dahlin, D. Dohi, M. Kanje, N. Terada, J. Hand Surg.: Br. Eur. Vol. 1997, 22, 299.

[45] M. A. Bisby, Fed. Proc. 1982, 41, 2307.

[46] L. S. Zweifel, R. Kuruvilla, D. D. Ginty, Nat. Rev. Neurosci. 2005, 6, 615.

[47] A. B. Ghnenis, R. E. Czaikowski, Z. J. Zhang, J. S. Bushman, J. Vis. Exp. 2018, 137. https://doi.org/10.3791/58031

[48] S. E. MacKinnon, A. L. Dellon, J. P. O’Brien, Muscle Nerve 1991, 14, 1116.

[49] E. C. Novosel, C. Kleinhans, P. J. Kluger, Adv. Drug Delivery Rev. 2011, 63, 300.

[50] P. Sierpinski, J. Garrett, J. Ma, P. Apel, D. Klorig, T. Smith, L. A. Koman, A. Atala, M. Van Dyke, Biomaterials 2008, 29, 118.

[51] H. K. Wang, Y. X. Wang, C. B. Xue, Z. M. Li, J. Huang, Y. H. Zhao, Y. M. Yang, X. S. Gu, Neural Regener. Res. 2016, 11, 168.

[52] H. Wang, H. Zhu, Q. Guo, T. Qian, P. Zhang, S. Li, C. Xue, X. Gu, Front. Cell. Neurosci. 2017, 11, 323.

[53] M. Blais, P. Levesque, S. Bellenfant, F. Berthod, Tissue Eng., Part A 2013, 19, 1655

[54] L. P. Yan, J. Silva-Correia, V. P. Ribeiro, V. Miranda-Goncalves, C. Correia, A. da Silva Morais, R. A. Sousa, R. M. Reis, A. L. Oliveira, J. M. Oliveira, R. L. Reis, Sci. Rep. 2016, 6, 31037.

[55] a) A. P. Balgude, X. Yu, A. Szymanski, R. V. Bellamkonda, Biomaterials 2001, 22, 1077; b) J. Bilsland, M. Rigby, L. Young, S. Harper, J. Neurosci. Methods 1999, 92, 75.

[56] a) C. Deister, C. E. Schmidt, J. Neural Eng. 2006, 3, 172; b) H. B. Wang, M. E. Mullins, J. M. Cregg, A. Hurtado, M. Oudega, M. T. Trombley, R. J. Gilbert, J. Neural Eng. 2009, 6, 016001.

[57] S. Wang, A. C. Wan, X. Xu, S. Gao, H. Q. Mao, K. W. Leong, H. Yu, Biomaterials 2001, 22, 1157.

[58] W. Chang, M. B. Shah, P. Lee, X. Yu, Acta Biomater. 2018, 73, 302. 